\title{
A novel test configuration design method for inverse identification of in-plane moduli of a composite plate under the PFEUM framework
}

\author{
M.Z. Siddiqui ${ }^{a}$, S.Z. Khan ${ }^{a, b}$, , M.A. Khan, M. Shahzad ${ }^{d}$, , K.A. Khan ${ }^{\mathrm{e}}$, S. Nisar ${ }^{\mathrm{a}}$ \\ ${ }^{a}$ Department of Engineering Sciences, PN Engineering College, National University of Sciences and Technology \\ (NUST), Karachi, Pakistan \\ ${ }^{b}$ Department of Mechanical Engineering, Faculty of Engineering, Islamic University of Madinah, Madinah, PO Box \\ 170, Kingdom of Saudi Arabia \\ 'School of Aerospace, Transport and Manufacturing, Cranfield University, Cranfield, UK \\ ${ }^{d}$ Space and Upper Atmosphere Research Commission (SUPARCO), Karachi, Pakistan \\ ${ }^{e}$ Department of Aerospace Engineering, Khalifa University of Science, Technology and Research (KUSTAR), Abu \\ Dhabi, UAE
}

*Corresponding Author

\section{Abstract}

We propose a novel sensitivity based approach which predicts and explains the accuracy of material parameter identification for a composite plate using the Projected Finite Element Update Method (PFEUM). A typical experiment using the PFEUM technique involves a plate specimen held at three or four supports and bent under the application of a point load. TwoDimensional Digital Image Correlation (2D-DIC) is used to measure the pseudo displacements resulting from the projection of out-of-plane deflection of the plate onto the image plane. A cost function relating the projected numerical and experimental displacement fields is then minimized to obtain the material parameters. It is shown that the contribution of a specific material parameter in the observed displacement field influences the accuracy of its identification. The contributions from material parameters are first quantified in terms of sensitivity criterion which may be tailored by changing the elements of test configuration like location of supports, the load application point and the specimen geometry. Several test configurations are designed by maximizing the sensitivities corresponding to individual material parameters. The relevance of proposed sensitivity criterion in these configurations is then 
validated through material identification in simulated experiments with added Gaussian noise. Finally, a thin CFRP plate is tested under these configurations to demonstrate the practical use of this approach. The proposed approach helps in robust estimation of the in-plane elastic moduli from a bent composite plate with a simple 2D-DIC setup without requiring measurement of the actual plate deflection or curvatures.

Keywords: 2D field measurements; Inverse identification; Finite Element Model Update method; Out-of-plane motion; Perspective projection; Parameter sensitivity; In-plane Elastic Moduli

\subsection{Corresponding author contact:}

Sohaib Zia Khan

Email: Sohaib.Khan@pnec.nust.edu.pk

Phone: +923332273100

Address: PG Building, PNEC, Habib Rehmatullah Road, National University of Sciences and Technology, Karachi, Pakistan 75350. 


\section{Introduction}

The cost benefit offered by laminated composites has brought about their widespread use in different industrial and household applications and has resulted in light weight products being produced at increasingly competitive costs. The weight reduction, however, also requires that the precise material response be known beforehand at the time of product design to avoid inservice failures. Characterization of the mechanical response of laminated composites presents unique challenges due to their anisotropic nature. In general, the displacement response of composites involves contributions from more than one material parameters [1]. The ASTM standards for characterization of laminated composites provide specific guidelines for specimen preparation and testing. Individual test guidelines, detailing the geometry and loading conditions, ensure that the displacement response from different material parameters are decoupled and a homogeneous displacement field is achieved which is mandatory when direct measurement of individual material parameters is sought [2]. These direct methods, however, are limited in their application as they require a large number of specimens and expendable strain gauges for complete characterization of material response [3].

The limitations present in the direct methods may be overcome if the test method is able to utilize heterogeneous displacement fields resulting in relaxation of the stringent geometry and loading requirements [4]. Recent research in this area has resulted in development of a range of inverse methods which precisely require the displacement response to be heterogeneous thus enabling identification of multiple material parameters from a single test. Contrary to the classical identification techniques where heterogeneous displacement or strain fields are a limiting condition, a variety of inverse methods are now available that make use of this heterogeneous information [5]. This paper deals with a particular application of an inverse method called the Finite Element Model Updating technique - also referred to as the Finite Element Update Method (FEUM) [6, 7].

The FEUM technique is applied using displacement [8] as well as natural frequencies $[9,10]$ for identification of material constitutive parameters. In a typical FEUM problem, a set of unknown constitutive parameters is assumed and using a FE model, a numerical displacement (or strain) field is generated. A similar displacement field is found by experimental measurement on the 
specimen whose material properties are to be determined. A cost function comprising of the displacement gap between the experimental and Finite Element (FE) solution of the surface response is then minimized in an iterative fashion to yield the material parameters being sought. [11]. Although full-field data on the whole surface of the specimen is desirable, the technique is equally applicable when the full-field data is available in only part of the domain [12].

Several researchers have adopted the FEUM based techniques for identification of material constitutive parameters in composite materials. For example Lecompte et al. [1] used cruciform shaped specimens made from glass fiber reinforced epoxy in biaxial tension. Bruno et al. [13] conducted study on the identification of elastic properties of unidirectional Graphite/PEEK using a plate specimen under flexural loading. In-plane properties of an orthotropic laminate were determined by Molimard et al. [14] using a thin plate specimen with a central hole loaded in tension. Wang and Kam [15] identified the elastic parameters of an orthotropic plate made from graphite/epoxy laminate. The rectangular plates were tested in a CCCC boundary condition (CCCC denotes clamping at all four edges, each $C$ representing an edge) and loaded with two types of loads: a point load at the center and a distributed pressure. A T-shaped specimen subjected to complex stress state was employed by Grédiac et al. [16] for identification of in-plane constitutive parameters. In addition to lab scale specimens, the inverse methods are also applicable to real structures. For example, in vivo characterization of anisotropic properties of human skin on a volar forearm was performed by Meijor et al. [17]. The FEUM approach was also used for identification of interlaminar fracture behavior of a unidirectional thermoset composite material by Mathieu et al. [18].

The tailoring of test configuration parameters has been studied earlier for design of an optimal test configuration for inverse identification of constitutive parameters in laminated composites. However, most of the earlier work in this direction relates to inverse identification using the Virtual Fields Method (VFM) [19] which relies on satisfaction of the global or weak form of equilibrium equations. The Finite Element Update Method (FEUM) has attracted little attention in this regard due to the absence of analytical relations to evaluate the sensitivity of identified parameters. The process of selection of optimized virtual fields for inverse identification yields noise sensitivity coefficients corresponding to the orthotropic stiffness's [20]. Pierron et al [21] 
have presented an optimization strategy which uses these sensitivity coefficients for constructing a cost function balancing the sensitivities of all material parameters in a composite specimen. The standard Unnotched Iosipescu (UI) test was used for the study with displacement measurements done by speckled interferometry. A similar cost function based on the global noise sensitivity coefficients normalized with respect to the maximum plate deflection have been used in $[3,22]$. The authors used grid method for measurement of curvatures in a bent composite plate specimen while the identification was done using the VFM. A numerical simulator for design of an optimal test configuration is presented by Rossi and Pierron [23] which is able to accurately model the parameters associated with the UI test. Under the VFM framework, the authors did a comprehensive study highlighting the effects of stress limit scaling, displacement field anisotropy, smoothing of strain field and the influence of missing data near the specimen boundary on parameter identification. In another research [24], the authors used this numerical simulator to study the effect of DIC settings in terms of identification error for selection of optimal test settings.

The displacement response of the specimen surface can also be captured by the optical techniques like Two- or Three-dimensional Digital Image Correlation (DIC) [25]. Thetwodimensional Digital Image Correlation (2D-DIC) measures the in-plane surface displacements by comparing two images taken before and after the surface deformation $[26,27]$. This method has benefits like ease of setup, less requirement of computation power as well as ease of calibration and low cost. In this method, a single camera is placed for taking images of the deforming surface in a way that the optical axis of the camera is parallel to the outward normal of the surface under consideration [28]. The specimens are tested with the 2D-DIC. They are generally either flat and subjected to in-plane loading (tension, compression, shear, biaxial or a combination of these loading conditions) $[29,30]$; or at least the surface under observation has predominantly in-plane deformation [31]. Because of the simplicity and other benefits that it offers, 2D-DIC is employed in a large number of applications [32]. The out-of-plane displacements cannot be detected and result in pseudo displacements seen as artificial strains in the DIC output $[33,34]$.

In plate bending experiments, the displacements are chiefly out-of-plane while the contribution of in-plane displacements is minimal. For such an arrangement, application of 2D-DIC results in 
measurement of a projected displacement field [29] which can only be utilized for identification of material constitutive parameters using a displacement gap based FEUM technique [30]. The Projected Finite Element Update Method (PFEUM) [35] relies on the minimization of a displacement gap between measured and computed displacement fields projected onto the image plane of camera. The primary advantage of this approach is that it allows use of a simple 2D-DIC setup for material identification using specimens undergoing three-dimensional deformation; thus, becomes cost effective and computationally reasonable. The details of the mathematical development of the PFEUM technique can be found in $[29,30]$.

In this research, we propose a novel sensitivity based method for design of a test configuration for material parameter identification using the PFEUM technique. The proposed method produces displacement fields with controlled contributions from the material parameters. The test setup is designed such that it allows alteration in the heterogeneity of the observed displacement field by controlling the setup parameters like position of supports, load location, fiber orientation and the aspect ratio. In the next section, a matrix based description of the equations of perspective projection developed earlier in [35] is given using the homogeneous coordinates. The inverse problem under the PFEUM framework is then reformulated. Later, sensitivity criterion for contribution of different material parameters in overall displacement field is derived using the FE simulations. At the end experimental data, for identification of in-plane elastic moduli of a CFRP plate, is presented to verify the validity of sensitivity criterion based on some tailored test configurations.

\section{Method}

\subsection{Projected Finite Element Update Method}

In this article, a compact matrix based description of the PFEUM technique is given which helps in the development of sensitivity criterion for design of test configuration and is conveniently applicable in a computer program. We start by considering the general case of projecting a point

in space $p(x, y, z)$ onto the image plane of an imaging system as shown in Fig. 1(a). A right handed Cartesian coordinate system is chosen to be parallel to the conventional image coordinate system with $\mathrm{y}$-axis pointing downwards. Let $v\left(0,0,-z_{0}\right)$ be a point away from the image plane which lies on the optical axis. The perspective projection of point $p$ on the image 
plane is the intersection of line $\overline{v p}$ with the image plane and is identified as $p^{\prime \prime}\left(x^{\prime \prime}, y^{\prime \prime}, z^{\prime \prime}\right)$. The point $v$ here is the center of perceptivity or viewpoint. The image plane normal, $n$, and the view direction are coincident with the z-direction.

A 2D version of Fig. 1(a) is shown in Fig. 1(b). Using similarity of triangles, we can directly write as

$\frac{y}{y^{\prime \prime}}=\frac{z_{0}-z}{z_{0}}=\frac{x}{x^{\prime \prime}}$

From where the position of projected point may be computed as

$x^{\prime \prime}=\frac{z_{0}}{z_{0}-z} x, y^{\prime \prime}=\frac{z_{0}}{z_{0}-z} y$

Since $z$ may be negative or positive, we can generalize these equations as

$x^{\prime \prime}=\frac{z_{0}}{z_{0}+z} x, y^{\prime \prime}=\frac{z_{0}}{z_{0}+z} y$

Equations (2) is similar to those derived earlier in [30] with $\Delta Z$ replaced by $z$. The equations were earlier derived for a deforming body with $\Delta Z$ being the out-of-plane displacement of a point initially lying on the image plane. However, the description presented here is more generic and is derived directly from the principles of projective geometry [36]. The current description allows projection of any point in space irrespective of its underlying deformation or loading history.

It is interesting to note that the camera model given by equations (2), which forms the basis of pinhole camera model [25], is nonlinear in $z$ and hence cannot be represented by simple matrix multiplication. To make the camera model linear, we can use the homogeneous coordinates [25] which represent points and lines in the projective space using vectors with four coordinates also called 4 vectors. Conversion from projective to Euclidian space is achieved by dividing the first three coordinates with the fourth coordinate which serves as a scaling parameter. From the geometry of perspective projection any point on the line joining points $p$ and $v$, and intersecting the image plane has coordinates of the form

$p^{\prime}=M p$

and, 
$M=n^{T} v+(n . v) I_{4}$

Where, $I_{4}$ is the $4 \times 4$ identity matrix. Equation (3) gives the linear form of the pinhole camera model and permits the perspective projection of a point to be taken by simple matrix multiplication. The perspective projection matrix $M$ is a linear transformation which maps points in the three-dimensional space to their corresponding projection points on the image plane. For the proof of equation (4) refer to [37].

Let $p(x, y, z, 1)$ and $v\left(0,0,-z_{0}, 1\right)$ be the homogeneous coordinates of the points $p$ and $v$ respectively, and let the image plane normal towards the viewpoint be represented by $n(0,0,-1,0)$ as shown in Fig. 1(a). Substituting the values in equation (4) gives

$M=\left[\begin{array}{cccc}-z_{0} & 0 & 0 & 0 \\ 0 & -z_{0} & 0 & 0 \\ 0 & 0 & 0 & 0 \\ 0 & 0 & -1 & -z_{0}\end{array}\right]$

And since $\lambda M p=\lambda p^{\prime}$, we can divide $\mathrm{M}$ by a scalar $\left(-z_{0}\right)$ to give

$M=\left[\begin{array}{cccc}1 & 0 & 0 & 0 \\ 0 & 1 & 0 & 0 \\ 0 & 0 & 0 & 0 \\ 0 & 0 & \frac{1}{z_{0}} & 1\end{array}\right]$

Substituting back in equation (3) gives

$\left\{\begin{array}{l}x^{\prime} \\ y^{\prime} \\ z^{\prime} \\ w\end{array}\right\}=\left[\begin{array}{llll}1 & 0 & 0 & 0 \\ 0 & 1 & 0 & 0 \\ 0 & 0 & 0 & 0 \\ 0 & 0 & \frac{1}{z_{0}} & 1\end{array}\right]\left\{\begin{array}{l}x \\ y \\ z \\ 1\end{array}\right\}$

Solving for $\left(x^{\prime}, y^{\prime}, z^{\prime}\right)$ and $w$ gives the homogeneous coordinates of the projection on image plane as

$x^{\prime}=x, y^{\prime}=y, z^{\prime}=0, w=\frac{z+z_{0}}{z_{0}}$

Where, $w$ is a scaling parameter used to convert homogeneous coordinates to Euclidian coordinates as 
$x^{\prime \prime}=\frac{x^{\prime}}{w}, y^{\prime \prime}=\frac{y^{\prime}}{w}$

Substituting for $w$ in equation (6) yields the Euclidian coordinates identical to those in equation (2). Hence the linear form presented in equation (3) gives the same results as equation (2) but is preferred because of the ease of application. The perspective projection matrix needs to be evaluated only once and thereafter only a simple matrix multiplication can be used to project a set of points from the three-dimensional space to the image plane. The hierarchy of projective transformations also allow multiple transformations to be concatenated in a single expression [38]. It is therefore possible to combine the rigid body motion of a body with subsequent projection in a single step as

$p^{\prime}=M C p$

Where, $C=[R t]$ is a $4 \times 4$ matrix that applies rigid body transformation to $p$ with $R$ and $t$ representing the rigid body rotation and translation respectively. The homogeneous coordinates are finally converted to Euclidian coordinates by dividing by the scaling parameter $w$.

In 2D-DIC, the surface under observation and its deformed configuration are assumed to lie within the image plane. This can be seen from equation (6). If the point $p$ in Fig. 1(a) lies on the image plane then $w=1$ and $x^{\prime \prime}=x, y^{\prime \prime}=y$. This means a point on the image plane projects onto itself and hence applying the linear transformation $M$ to points on the image plane does not introduce any change in point positions. However, when the specimen deforms out-of-plane or when there is relative rigid-body motion between the camera and specimen, the deformed surface is projected by the linear transformation given by matrix $M$ and subsequent DIC computations are performed on the projected image.

Now consider a plate specimen pinned at three points $(S 1, S 2$ and $S 3)$ and acted upon by a force $F$ as shown in Fig. 2 . The specimen is initially aligned with the image plane $\Gamma$ and deforms outof-plane under the application of load. Let $X$ be a set of points defining the specimen surface before deformation. Now $X^{\prime \prime}$, the projection of $X$ onto the image plane $\Gamma$, is identical with $X$ as the surface is initially in-plane. Let the coordinates of points on the deformed surface be $\tilde{X}$. In the presence of rigid-body motion, the points on the deformed surface assume the positions $C \tilde{X}$; 
where $C=[R t]$ is the rigid body transformation matrix. The projection of transformed deformed surface on $\Gamma$ is then given by

$\widetilde{X}^{\prime}=M C \tilde{X}$

$\widetilde{X}^{\prime}$ can be converted to Euclidian coordinates $\widetilde{X}^{\prime \prime}$ by using equation (6). The experimental displacement field determined through 2 D-DIC is

$u_{e}=\tilde{X}^{\prime \prime}-X^{\prime \prime}$

Note that the coordinates $\tilde{X}^{\prime \prime}$ and $X^{\prime \prime}$ in equation (9) are Euclidean (3 vectors) since addition is not defined among point coordinates in the perspective space. The double prime with $X^{\prime \prime}$ is retained to indicate the operation in Euclidian coordinates. Since the displacement field computed by $2 \mathrm{D}-\mathrm{DIC}$ is already on the image plane, $\tilde{X}^{\prime \prime}$ in equation (9) need not be calculated separately and $u_{e}$ is measured directly by 2D-DIC and includes the effect of sample deformation as well as rigid-body out-of-plane motion. Similarly, the numerical displacement field, found using FE analysis of the specimen under the same load and boundary conditions, may be found by the linear transformations of deformed coordinates of FE solution $\tilde{X}_{f}$ as

$$
\left.\begin{array}{c}
X_{f}^{\prime}=X_{f} \\
\tilde{X}_{f}^{\prime}=M C \tilde{X}_{f} \\
u_{f}=\tilde{X}^{\prime \prime}{ }_{f}-X^{\prime \prime}{ }_{f}
\end{array}\right\}
$$

Where, in the third of equations (10), $X^{\prime \prime}{ }_{f}$ and $\tilde{X}^{\prime \prime}{ }_{f}$ are the Euclidian equivalents of $X^{\prime}$ and $\tilde{X}_{f}^{\prime}$ respectively. In equation (10) it is assumed that prior to the load application the plate specimen lies perfectly in-plane with its outward normal aligned with the optical axis. In case this assumption is not valid, the undeformed or initial coordinates $X_{f}$ may need to be transformed by a different transformation matrix as

$X_{f}^{\prime}=M C_{0} X_{f}$

Where, $C_{0}$ is the rigid-body transformation matrix that aligns the FE model with the plate specimen. Finally, a cost function may be defined on $\Gamma$ which relates the experimental and numerical displacement fields as 
$S(c)=\left[u_{e}-u_{f}\right]^{T}\left[u_{e}-u_{f}\right]$

The cost function defined by equation (12) is a function of material parameters $c$ and is minimized in an iterative fashion to seek an optimal set of material parameters. The overall inverse problem is then formulated as

$\min _{c} S(c) \quad c \in C$

Where, $C$ is the set of all permissible values of constitutive parameters satisfying the governing equation. If equation (10) is used to estimate the numerical displacement field, an additional six parameters specifying the rigid body modes may be incorporated to account for small relative motion between camera and specimen. The minimization will yield the correct constitutive parameters only if the specimen deformation produces a projected displacement field that is unique from those introduced by the general rigid-body motion of the specimen $[29,30]$. The schematic of the identification process is shown in Fig. 3.

\subsection{Sensitivity Criterion}

For a transversely isotropic laminate, there are four independent material parameters that uniquely define the displacement response. These four independent material parameters are the fiber direction modulus $E_{1}$, the transverse modulus $E_{2}$, the in-plane Poisson's ratio $v_{12}$ and the in-plane shear modulus $G_{12}$. We denote the numerical projected displacement field that is obtained by a given set of independent material parameters, geometry, load and boundary conditions as $u_{0}$ which, in the absence of rigid-body motion, is obtained from equation (9) as

$u_{0}=\tilde{X}^{\prime \prime}{ }_{0}-X^{\prime \prime}$

Where, the matrix $X^{\prime \prime}$ represents the point coordinates in the undeformed configuration while $\tilde{X}^{\prime \prime}{ }_{0}$ is the matrix containing coordinates of the corresponding deformed configuration. $u_{0}$ may be termed as the nominal displacement field which serves as the reference. Now, if one of the four parameters is given a small increment, the displacement field thus obtained will be modified in proportion to the contribution of that parameter in the nominal displacement field. Thus

$u_{\mathrm{i}}=\tilde{X}^{\prime \prime}{ }_{\mathrm{i}}-X^{\prime \prime}$ 
And,

$d_{i}=\operatorname{abs}\left(u_{0}-u_{i}\right)$

Where, $d_{i}$ is the absolute difference between the nominal and modified displacement fields corresponding to the $i$ th incremented material parameter. Similar incremental displacement fields may be obtained for all material parameters. We define a matrix $d$ to contain the incremental displacement fields from all material parameters in individual columns as.

$d=\left[\operatorname{vec}\left(d_{1}\right) \operatorname{vec}\left(d_{2}\right) \operatorname{vec}\left(d_{3}\right) \operatorname{vec}\left(d_{4}\right)\right]$

Where, $d_{i}, i=1$ to 4 , represent the incremental fields for $E_{1}, E_{2}, \vartheta_{12}$ and $G_{12}$ respectively. The vec operator converts $d_{i}$ into a single column vector. If there are $n$ points in the displacement field, vector $d_{i}$ has the size $2 n \times 1$ with first $n$ elements containing the $x$ coordinates while the next $n$ elements containing the $y$ coordinates of the incremental displacement field. A criterion defining the sensitivity of the overall displacement field towards individual parameters may now be defined as

$s c_{r e l}=\frac{\sum_{i}\left(d_{i j}\right)}{\sum_{i} \sum_{j}\left(d_{i j}\right)} * 100$

$s c_{r e l}$ is a $4 \times 1$ row vector containing the percent relative change in the nominal displacement fields due to small increments in the individual material parameters. The elements of $s c_{r e l}$ range between 0 and 100 and $s u m\left(s c_{r e l}\right)$ is always equal to 100 . The parameter with highest value of $s c_{r e l}$, therefore, would be expected to dominate the displacement response of the test specimen and would be easily identifiable due its distinct signature. On the other hand, a parameter with the lowest $s c_{r e l}$ value would be very prone to underlying noise and difficult to identify. In an ideal case, therefore, all four material parameters should have a $s c_{r e l}$ value of 25 . The next section gives a method to design a test configuration based on $s c_{r e l}$ as sensitivity criterion.

\subsection{Design of test configuration}

This section presents a process of sensitivity based design of the test configuration for a plate bending experiment under PFEUM framework. A parametric description of the plate bending specimen is given which is useful in formulating the optimization problem for tailoring of measured displacement fields. Consider a rectangular composite plate with length $L$ and width 
$W$ as shown in Fig. 4. A right handed Cartesian coordinate system is placed at the center of plate which is aligned with the image coordinate system. The $Z$ axis is coincident with the optical axis and points away from the camera. The fiber orientation $\alpha$ is zero parallel to the $\mathrm{X}$ axis and increases in the counter-clockwise direction. The outer plate edges are marked with solid lines. The plate is held in place by pin supports which do not restrict plate rotation. The supports marked $S 1, S 2, S 3$ and $S 4$ can be positioned along the edges on the inner boundary marked by dotted lines. The inner boundary is offset from the outer boundary by distances of $b x$ and by in $x$ and $y$ directions respectively as shown in Fig. 4 . The four corners are marked $C 1$ to $C 4$ in the counter-clockwise direction. The position of supports is defined in a normalized boundary coordinate system (BCS) defined at each corner. As shown for $C 1$, the BCS is defined such that the support location $S 1$ is 0 at $C 1$, positive between $C 1$ and $C 2$ with a maximum value of 1 at $C 2$, and negative between $C 1$ and $C 4$ with a minimum value of -1 at $C 4$. In a similar fashion all supports can move between -1 and +1 along the inner boundary edges connecting the corresponding corner. The load application point has coordinates $L x$ and $L y$ which are also normalized along the $x$ and $y$ directions. $L x$ is -0.5 at the left edge and +0.5 at the right edge while $L y$ is -0.5 at the top edge and +0.5 at the bottom edge. The direction of load is towards the camera such that plate deformation is primarily out-of-plane.

In total there are 9 independent geometric parameters that completely define the test configuration. These parameters may be gathered in a set such that

ip $=\{L, W, L x, L y, \alpha, S 1, S 2, S 3, S 4\}(21)$

With the plate completely defined, a simple optimization may be carried out to maximize the sensitivity criterion $s c_{r e l}$ by tuning a subset of the geometric parameters in ip. The constraints on geometric parameters during optimization are dictated by specimen manufacturing considerations and dimensions of test bench. The overall theme of the process of optimizing a test configuration is depicted in Fig. 5. Alternatively, the optimization may be skipped and a manual tuning of geometric parameters may be carried out where in a small subset of $i p$ is manually modified to attain the required contribution from a specific material parameter. 


\section{Experimental setup}

The experimental setup used for inverse identification in our earlier work [35] consisted of a stainless-steel test bench with $10 \mathrm{~mm}$ thick brackets for holding the specimen. The support locations were fixed at the corners and hence could not be altered. A redesigned test bench, Fig. 6, was used in present study which allows adjusting the support positions along the left and right edges of the specimen. The bench was fitted with leveling screws which were used along with the level gauge to align the outward normal of the specimen in a horizontal plane. The thickness of brackets has been increased to $20 \mathrm{~mm}$ resulting in a reduction in available specimen width to a maximum of $185.6 \mathrm{~mm}$.

The specimen was held in position between pointed bolts at each support location. These supports were modelled in FEA by holding a single node at the support location. The concentrated load on the back face of the specimen was applied by controlled rotation of a nut on $5 \mathrm{~mm}$ diameter threaded bolt. The arrangement was such that the rotation of nut caused the bolt to travel towards the plate. The magnitude of applied load was measured by an S-type FUTECK load cell with a maximum capacity of $45 \mathrm{~N}$ in compression.

The CFRP plates for the experiments were made using unidirectional tape of Carbon Fiber prepregs with epoxy resin. Detailed fabrication method and reference material properties $\left(E_{1}=\right.$ $145 \mathrm{MPa}, E_{2}=8.0 \mathrm{MPa}, v_{12}=0.31$ and $G_{12}=5 \mathrm{MPa}$ ) of the plates are given in [35]. All specimens tested in this work have dimensions of $185.6 \mathrm{~mm} \times 137 \mathrm{~mm}$. The speckle pattern for Image Correlation was printed on a 70 gsm white paper and pasted on the specimen surface. The pattern was developed in MATLAB using a grid with a spacing of $1 \mathrm{~mm}$ and a speckle size of 0.3 $\mathrm{mm}$ (0.023 pixels). A $0.2 \mathrm{~mm}$ (0.015 pixels) random error was introduced in the position of grid points in both $X$ and $Y$ directions to prepare a random pattern. Support locations as well as the center lines, shown in Fig. 7, were also printed to assist in the positioning of supports and alignment of specimen.

The camera used for image acquisition was a SONY XCG5005E monochrome camera with an image depth of 12 bits. The imaging sensor had an effective resolution of $2448 \times 2048$ pixels. The camera was fitted with a Schneider Optics high resolution lens having a variable focal length of 
$1.8 \mathrm{~mm}$ to $35 \mathrm{~mm}$. The perpendicularity of camera optical axis with specimen surface was ensured by carefully leveling the camera using a level gauge and then aligning an image grid with the specimen center lines and a similar grid pattern drawn on the back wall facing the experimental setup. The images were calibrated by using specimen width along the centerline.

The experimental displacement field was measured by analyzing the captured images with 2DDIC using an indigenously developed code OSM. Detailed description of the code, along with test results with standard images provided by Society for Experimental Mechanics, is given in [51, 57, 58]. Due to very small expected magnitude of the measured displacements, five images were taken at each load step and then averaged to minimize the measurement noise due to various effects such as light intensity and main frequency variations. The DIC grid had a spacing of 30 pixels with a subset size of 31 pixels. Low levels of expected deformation gradients allowed selection of relatively large grid spacing to reduce computation cost during DIC as well as cost function estimation. Under these conditions, the image correlation system gives a standard deviation of less than 0.01 pixels or $1 \mu \mathrm{m}$ when consecutive images without any deformation are compared. Since this noise is expected to be present in all experimental results, a random noise with a constant standard deviation of $1 \mu \mathrm{m}$ was added to all simulated displacement fields during the sensitivity study discussed in the next section.

\section{Results and discussion}

\subsection{The Finite Element model}

The FE model of the plate was made with ANSYS APDL script. The supports were modelled by suppressing translational degrees of freedom (DOF) at the support nodes. The presence of FE nodes at the supports and at the load application point were ensured by creating hard points

on these locations. The element coordinate system was oriented such that the fibers were aligned length wise in the $X$ direction.

The FE model of the plate was also validated experimentally. A composite specimen of $2 \mathrm{~mm}$ thickness was held in case 1 configuration. A linear displacement transducer (LDS) was used to measure the deflection of plate at its center as shown in Fig. 8. An initial load of $4.3 \mathrm{~N}$ was given and plate deflection was measured from $14 \mathrm{~N}$ to $32 \mathrm{~N}$ with $2 \mathrm{~N}$ steps. The plate was modelled 
using the 20-node SOLID186 brick elements with an edge length of $5 \mathrm{~mm}$ and as a single element across the plate thickness. For comparison, the 8-node SHELL281 elements were also used for plate modelling with $5 \mathrm{~mm}$ element size. In order to extract surface displacements and apply supports on correct plate surface, the shell section was offset to element BOT plane which corresponds to the plate surface facing the camera and where the supports physically touch the plate.

The Figure 9 shows the comparison of the experimentally measured plate deflection with the FEA results. It is clearly seen that the deflection with both solid and shell elements closely matches the experimental deflection. The solid elements were preferred even the choice between the shell and the solid seems arbitrary. The choice was made to ease possible integration with the support structure made from the solid elements. When nonlinear geometric effects were enabled, the plate deflection was over-predicted at all loads. The disparity in between the linear and the non-linear solution is apparently in disagreement with the large deflection theory. It would predict lower plate deflection due to stress stiffening. The difference is probably because of the presence of the supports is on the surface of the plate instead on the mid-plane. As the plate deflects, the mid-plane is relatively free to stretch and resulting an increase in the plate deflection. The disparity could be removed if the rotation at supports were suppressed for both the solid and the shell elements. For example, if the plate was held between the two pointed bolts at each support location (i.e.one on each face of the plate). The rotation at supports would be suppressed and the stretching of mid-plane would be restricted. The linear and nonlinear solutions for this support condition agree but do not match with the experimental results for this setup. The detail investigation of this bias due to the nonlinear effects is beyond the scope of this work and will be reported in a future publication. The nonlinear effects were ignored in the presented research.All the test cases were designed to keep the maximum plate deflection at less than half of the plate thickness which kept the nonlinear effects to a minimum.

\subsection{Test configurations}

Five test configurations were tested in this work. Each of them was designated with a unique case number. The first two cases related to the configurations tested in [35] with the parameters converted to those of Fig. 4. Two more configurations having dominant contributions from $E_{1}$ 
and $G_{12}$ and a third configuration with balanced contributions from $E_{1}, E_{2}$ and $G_{12}$ have been designed in this work. The design was carried out on the available specimen geometry. Hence the specimen dimensions and fiber orientation were not changed. Table 1 shows the geometric parameters for all the test cases.

The sensitivity criterion $s c_{r e l}$ for each configuration was estimated using a simulated displacement field generated through a FE analysis and then projected using the equation (11). A constant load of $24 \mathrm{~N}$ (corresponds to the average load applied during the identification experiments) was used for each simulation. The sensitivity criterion corresponding to the five test cases for each material parameter as well as the maximum plate deflection at this load are given in Table 2. There were two conditions imposed during the design of the new test cases. The maximum plate deflection was kept less than half of the plate thickness to keep the nonlinear effects minimum; and the minimum distance between any two supports was set as $22 \mathrm{~mm}$ which was the minimum physical distance achievable between the support points (i.e. when two support brackets in physical contact to each other).

Case 1 related to a test configuration with four supports at the corners and a point load at the plate center. Table 2 indicates that the maximum contribution in the displacement field obtained from $E_{2}$ with a $s c_{r e l}$ value of $74.3 \%$. While $v_{12}$ had the minimum contribution with $s c_{r e l}=$ $3.0 \%$. Thus case 1 was expected to show best convergence of $E_{2}$.

The contribution of $E_{1}$ and $G_{12}$ were more pronounced in case 2, with only three support points, as indicated by sensitivity values of $37.6 \%$ and $36.0 \% . E_{2}$ had a slightly lower value of $s c_{r e l}=$ $23.8 \%$ while $v_{12}$ contributed very weakly as depicted by a small value of $s c_{r e l}=2.7 \%$.

As discussed earlier, the sensitivity criterion may be tailored for a specific parameter by careful adjustment of geometric parameters. The three new test configurations analyzed in this work were based on variation of sensitivity criteria due to change in different test parameters. Figures 10 thru 14 show the variation of sensitivity criteria due to change in selected test parameters. For example, Fig. 10 shows the effect of moving $L X$ from the left to right edge of the plate keeping all other parameters fixed as in case 1 . It is seen that the contribution from $E_{2}$ and $v_{12}$ remain almost unchanged while E1 and G12 reach their minimum and maximum values, 
respectively, as the load application point approaches the plate center. The most dominant contribution from $E_{2}$ is thus found around $L X=0$ which corresponds to case 1 configuration.

If instead of $L X, L Y$ is varied from minimum to maximum - from top to bottom edge - the contribution from $E_{1}$ and $E_{2}$ both change rapidly with $E_{1}$ being maximum as the top and bottom edges as shown in Fig. 11. From this figure, an $E_{1}$ dominant configuration was selected and designated as case 3 with the load applied midway between $S 2$ and $S 3$. Here the maximum resistance to deformation is generated due to the fiber direction modulus $E_{1}$ with a high contribution of $65.2 \%$ while the sensitivity of $E_{2}$ drops rapidly towards the edges. The next highest contribution comes from $G_{12}$ which has $s c_{r e l}=26.1 \%$, while $E_{2}$ and $v_{12}$ have contributions of $5.6 \%$ and $3.2 \%$ respectively.

Figure 12 plots the variation in material parameter contributions as load is moved along the bottom edge from left to right in case 3 configuration. As expected, $E_{1}$ remains dominant throughout as the load remains directly at the fibers going from one support to the other. Furthermore, no significant change in the contribution from all other parameters is seen.

Figure 13 plots the effect of moving $S 3$ in case 1 configuration from bottom to top along the right edge of the plate. Although $v_{12}$ does not show any significant improvement, the sensitivities for all other material parameters change significantly. The sensitivities of $E_{1}$ and $E_{2}$ reach their maximum and minimum values, respectively, between $S 3=0.60$ to 0.70 while the sensitivity of $G_{12}$ continues to increase as the support $S 3$ is moved towards $S 4$.

Although the fiber orientation was constraint due to specimen availability, the effect of varying it from the reference $0^{\circ}$ in case 1 configuration was also studied. Figure 14 shows the variation of material parameter sensitivities due to change in fiber orientation in the plate specimen. As the fiber angle increases, the sensitivities of $E_{1}$ and $E_{2}$ move in the opposite directions and around $35^{\circ}$ to $40^{\circ}$, the contribution from $E_{1}$ becomes maximum while $E_{2}$ reaches a minimum value. This corresponds to a point when the load is applied directly on fibers aligned between the two support points ( $S 2$ and $S 4$ in this case). This result is consistent with the plot of Fig. 11, where the contribution of $E_{1}$ is most dominant when the load is applied on the top or bottom edge. The 
sensitivity of $G_{12}$ is maximum at about $50^{\circ}$ fiber orientation while $v_{12}$ remains almost unaffected by change in fiber orientation.

From Fig. 10 thru Fig. 14, it is clearly seen that the contribution of in-plane Poisson's ratio $v_{12}$ remains unaffected and does not depend strongly on any single parameter including the fiber orientation. Hence a $v_{12}$ dominant configuration could not be found through an intuitive tailoring of test configuration. The best point for a $G_{12}$ dominant configuration appears to be when $S 3$ is above 0.7 as shown in Fig. 13. However, a check on maximum plate deflection, Fig. 15, shows that the plate deflection rapidly increases above $S 3=0.6$ and exceeds the half plate thickness criterion. Moreover, the distance between supports $S 3$ and $S 4$ becomes less than $22 \mathrm{~mm}$ when $S 3$ is greater than 0.8 . Since $G_{12}$ shows clear dependence on the position of load application point, Fig. 10 and Fig. 11, and the support position, Fig. 13, an optimization was run with $L x, L y, S 1$ and $S 4$ as independent variables while keeping other geometric parameters fixed. Only four geometric parameters were selected to keep the design space as small as possible. The sensitivity criterion for $G_{12}$ was set as objective function with constraints on the minimum support distance and the plate deflection as discussed earlier. The configuration obtained through this optimization study, designated as case 4 , has maximum contribution of $50.4 \%$ from $G_{12}$, while $E_{2}$ also has a healthy contribution of $38.1 \%$.

Careful comparison of Fig. 11 and Fig. 13 indicates the possibility of finding a configuration with balanced contributions from $E_{1}, E_{2}$ and $G_{12}$. As $S 3$ reaches a value of about 0.45 , the contribution from these parameters tend to converge, as shown in Fig. 13, with decreasing contribution from $E_{2}$ and increasing contributions from $E_{1}$ and $G_{12}$. Since the same trend is observed as load moves downwards, increasing $L Y$ in Fig. 11, the two geometric parameters were tuned together to reach a point where $E_{1}, E_{2}$ and $G_{12}$ have almost equal contributions in the resulting displacement field as indicated by $s c_{r e l}$ values of $31.4 \%, 32.5 \%$ and $33.4 \%$ respectively. The test configuration thus obtained was designated as case 5.

In general, $E_{1}$ appears to dominate the displacement field when the load application point is near either the top or the bottom edge (the edge parallel to the fiber direction). If the load is applied at the center of the top or bottom edge (case 3), maximum resistance to deformation comes 
directly from the bending of the plate along the fiber direction and hence the fiber direction modulus plays the key role while the contribution from $E_{2}$ stays minimum.

The contribution from $E_{2}$, on the other hand, increases as the load application point moves away from the top and the bottom edges. The maximum contribution from $E_{2}$ will be apparent when the load application point is placed at the center of the left or right edge. At this location, the bending of plate in the transverse fiber direction is the main driving factor and maximum contribution is come from matrix deformation. As expected, the contribution form $E_{1}$ is minimum in this configuration. $G_{12}$ appears to be minimum for a perfectly symmetric configuration (case 1) and increases whenever the symmetry is disturbed by moving the load or any single support from its position in the case 1.

In all the test cases, it was noted that $v_{12}$ is least sensitive to any change in the geometric parameters and hence the most difficult parameter to identify. No configuration could be found, manually or through optimization, which had a dominant contribution from $v_{12}$ in the resulting displacement field. For this reason, it was decided to study the relationship between the proposed sensitivity criterion and the identification accuracy for the other three parameters.

It may be noted here that the $E_{1}$ and the $E_{2}$ dominant test configurations of case 3 and case 1 as well as the balanced configuration of case 5 could be achieved equally well through a numerical optimization but the approach presented here is more intuitive and helps in developing a thorough understanding of the effect of different geometric parameters on material parameter sensitivities. Furthermore, the present study was confined to obtain variations of case 1 having four supports. Alternatively, Fig. 10 thru Fig. 14 may be reproduced for a plate held at only three points to obtain the variations of case 2 . In the next section, the results of material parameter identification though simulated as well as real experiments for all five cases are presented and discussed.

The test cases were initially analyzed using simulated experiments wherein a simulated displacement field, projected using equation (11), with an added random noise with standard deviation of $1 \mu \mathrm{m}$, was used as experimental data for inverse identification. Since no rigid body error was introduced during generation of simulated displacement field, only the four material 
parameters were used as independent variables during the minimization of cost function in equation (13). The convergence bounds were set as $\pm 75 \%$ of the target values. Since the convergence accuracy is not affected by the initial guess [35], the target values were passed as initial guess to speed up the convergence process. The simulation was repeated for each case using 10 load values ranging from $15 \mathrm{~N}$ to $33 \mathrm{~N}$ with $2 \mathrm{~N}$ steps and an initial load of $5 \mathrm{~N}$. The coefficient of variation $C O V$ and the relative error $E R R$ were estimated using the converged parameter values from all load steps as follows

$$
\begin{aligned}
& C O V=\frac{\text { standard deviation }}{\text { mean }} \times 100 \\
& E R R=\frac{\text { abs(mean-target value })}{\text { target value }} \times 100
\end{aligned}
$$

To quantify the ability of a given case to produce reliable parameter estimates a unified convergence criterion was defined by combining the $C O V$ and $E R R$.

$C C=\sqrt{\left(C O V^{2}+E R R^{2}\right)}$

From our earlier work [35], where $C O V$ was used to ascertain successful convergence, it was found that for some cases the parameter estimates end up near the upper or lower bound for each load step hence giving a very low COV despite poor convergence as evident from a high value of $E R R$. However, the convergence criterion defined by equation (25) combines the effect of parameter variation from a mean value as well as the departure of mean from the target. Thus, a high value of $C C$ indicates poor convergence from either one or both of $C O V$ and $E R R$. Table 3 shows the three convergence estimators for the simulated experiments.

Finally, the test cases were verified by identification experiments using $2 \mathrm{~mm}$ thickness CFRP plate specimen tested in each of the five test configurations. The experimental displacement field (projected) was measured using 2D-DIC. Each experiment was repeated ten times at loads ranging from $15 \mathrm{~N}$ to $33 \mathrm{~N}$ with $2 \mathrm{~N}$ intervals and an initial load of $5 \mathrm{~N}$. During the identification step, all six rigid body modes along with the four material parameters were as variables for optimization. A sample comparison of the numerical and the experimental displacement fields at 
the end of identification process is given in Fig. 16 for the case 3 configuration at a load of $23 \mathrm{~N}$. In order to make the comparison, the numerical displacement field is first corrected for the rigidbody modes, recovered during the identification process, before a projection is taken.

Tables 4 to 8 give the detailed identification results for the five test cases indicating the estimated material parameters as well as the rigid body modes extracted in each test. The first three modes represent the in-plane rigid body motion while the last three modes correspond to the out-ofplane motion. The estimated $C O V, E R R$ and $C C$ from this experimental data are shown in Table 9. Figure 17 presents plot of the convergence criterion $C C$ against the sensitivity criterion $s c_{r e l}$ for simulated as well as experimental results. The figure shows a combined plot of convergence criterion for the four material parameters in all five test cases. It is clearly seen that as the value of $s c_{r e l}$ for any parameter increases, the convergence is steadily increased. Particularly for $s c_{r e l}$ greater than 30 very good convergence is observed. Since $s c_{r e l}$ for the in-plane Poisson's ratio $v_{12}$ is always less than 5 , the estimation of $v_{12}$ remains a challenge.

A comparison of simulated and experimental data shows that the experimental results have somewhat poor convergence compared to the simulated results for the same sensitivity values. This difference may be due to the unaccounted variations in the specimen thickness as well as the behavior of supports. Particularly the assumption of rigid supports is worth considering. In all five identification experiments, the extracted rigid body modes show considerable our-of-plane displacement as well as rotation about $x$ which could only arise if there was some unaccounted displacement of the specimen at the supports. A plot of the convergence criteria for simulated and experimental results were normalized with respect to the maximum criteria value as shown in Fig. 18. The overall trend of simulated and experimental data matches very well and confirms the validity of sensitivity criterion $s c_{r e l}$ as a valuable tool for design of test configuration under a PFEUM framework

The correct estimation of $v_{12}$ remains a challenge. From a total of 50 attempts at the estimation of $v_{12}$, the estimation was successful only in the first four load steps in case 1 and the second step in case 4 and case 5 . The criterion defined in this work is based on relative contributions of material parameters in the displacement fields. The results however indicate that a relative 
criterion may not be sufficient for estimation of a parameter which has small contribution in the overall displacement field. Another possible reason for the failure in estimation of $v_{12}$ is the relative magnitude of noise present in the measured displacement field compared to the contribution of $v_{12}$ in the overall displacement field.

Indirectly, the proposed criterion indicates the change in cost function, equation (12), that would result when a small change in the individual material parameters is introduced. Hence the material parameter with relatively high value of $s c_{r e l}$ is expected to show good identifiability whereas a parameter with lowest value of $s c_{r e l}$ value will be difficult to identify as its response will be eclipsed by the underlying measurement noise.

\section{Conclusions}

A systematic approach towards design of test configuration for inverse identification of material constitutive parameters in transversely isotropic laminates is presented for a plate bending experiment. First a compact matrix based description of the PFEUM technique is given. The use of homogeneous coordinates helps in converting the non-linear pin hole camera model to a linear form which is then utilized to reformulate the PFEUM equations.

A parametric description of the plate bending experiment is given which shows that there are nine independent geometric parameters that fully define the test geometry and may be tuned to control the composition of the overall displacement field of the specimen.

Sensitivity criterion based on the relative contribution of material parameters towards the overall displacement field is derived. Several test configurations have been picked which indicate the application of the proposed sensitivity criterion. The dominance of individual material parameters in displacement fields for the selected configurations is then explained in detail. It is observed that the contributions from $E_{1}, E_{2}$ and $G_{12}$ can be easily controlled by modification in only a small subset of the independent parameters. However, $v_{12}$ shows little sensitivity towards any of the independent parameters and hence no configuration could be identified which contained dominant contribution of displacement field from $v_{12}$. 
Simulated experiments containing white noise have been performed to verify the performance of each test configuration. The simulations indicate a clear relationship between the sensitivity criterion and the success of parameter estimation. Finally, this result is further validated from experiments performed with CFRP plate specimens. The experiments thus performed illustrate practical importance of the sensitivity criterion. The fifth test configuration, design for a balanced contribution from $E_{1}, E_{2}$ and $G_{12}$ gave the best parameter estimates for these in-plane elastic moduli.

The disparity between simulated and experimental results, observed in terms of comparatively poor convergence in experimental test results for the same sensitivity criterion has been explained. Uncontrolled parameters like specimen thickness variation, support deformations as well as the possibly high magnitude of signal-to-noise ratio appear to be the major factors affecting the accuracy of experimental results. An improved sensitivity criterion incorporating the absolute contribution from different material parameters in the observed displacement fields is proposed for future study to overcome the difficulty associated with the identification of $v_{12}$ by improving the signal-to-noise ratio.

\section{References}

1. Lecompte D, Smits A, Sol H, et al (2007) Mixed numerical-experimental technique for orthotropic parameter identification using biaxial tensile tests on cruciform specimens. International Journal of Solids and Structures 44:1643-1656 . doi: 10.1016/j.ijsolstr.2006.06.050

2. Pierron F, Alloba E, Surrel Y, Vautrin A (1998) whole-field assessment of the effects of boundary conditions on the strain field in off-axis tensile testing of unidirectional composites. Composites Science and Technology 58:1939-1947 . doi: 10.1016/S0266-3538(98)00027-X

3. Syed-Muhammad K, Toussaint E, Grédiac M (2009) Optimization of a mechanical test on composite plates with the virtual fields method. Struct Multidisc Optim 38:71 . doi: 10.1007/s00158-0080267-y

4. Grédiac M (2004) The use of full-field measurement methods in composite material characterization: interest and limitations. Composites Part A: Applied Science and Manufacturing 35:751-761 . doi: 10.1016/j.compositesa.2004.01.019

5. Pagnacco E, Moreau A, Lemosse D (2007) Inverse strategies for the identification of elastic and viscoelastic material parameters using full-field measurements. Materials Science and Engineering: A 452-453:737-745 . doi: 10.1016/j.msea.2006.10.122 
6. Rahmani B (2014) In-Situ Mechanical Properties Identification of Composite Materials Using Inverse Methods Based on Full-Field Measurements. École Polytechnique de Montréal

7. Kavanagh KT, Clough RW (1971) Finite element applications in the characterization of elastic solids. International Journal of Solids and Structures 7:11-23

8. Fazzini M, Dalverny O, Mistou S (2011) Identification of Materials Properties Using Displacement Field Measurement. Key Engineering Materials 482:57-65 . doi:

10.4028/www.scientific.net/KEM.482.57

9. Türker T, Bayraktar A (2008) Structural parameter identification of fixed end beams by inverse method using measured natural frequencies. Shock and Vibration 15:505-515

10. Xia Y, Hao H, Brownjohn JM, Xia P-Q (2002) Damage identification of structures with uncertain frequency and mode shape data. Earthquake engineering \& structural dynamics 31:1053-1066

11. Cooreman S, Lecompte D, Sol H, et al (2008) Identification of Mechanical Material Behavior Through Inverse Modeling and DIC. Experimental Mechanics 48:421-433 . doi: 10.1007/s11340007-9094-0

12. Grediac M, Hild F (2012) Full-Field Measurements and Identification in Solid Mechanics. John Wiley \& Sons

13. Bruno L, Felice G, Pagnotta L, et al (2008) Elastic characterization of orthotropic plates of any shape via static testing. International Journal of Solids and Structures 45:908-920 . doi: 10.1016/j.ijsolstr.2007.09.017

14. Molimard J (2005) Identification of the Four Orthotropic Plate Stiffnesses Using a Single Open-hole Tensile Test. Experimental Mechanics 45:404-411 . doi: 10.1177/0014485105057757

15. Wang WT, Kam TY (2000) Material characterization of laminated composite plates via static testing. Composite Structures 50:347-352 . doi: 10.1016/S0263-8223(00)00112-4

16. Grédiac M, Pierron F, Surrel Y (1999) Novel procedure for complete in-plane composite characterization using a single T-shaped specimen. Experimental Mechanics 39:142-149

17. Meijer R, Douven LFA, Oomens CWJ (1999) Characterisation of Anisotropic and Non-linear Behaviour of Human Skin In Vivo. Computer Methods in Biomechanics and Biomedical Engineering 2:13-27 . doi: 10.1080/10255849908907975

18. Mathieu F, Aimedieu P, Guimard J-M, Hild F (2013) Identification of interlaminar fracture properties of a composite laminate using local full-field kinematic measurements and finite element simulations. Composites Part A: Applied Science and Manufacturing 49:203-213 . doi: 10.1016/j.compositesa.2013.02.015

19. Guélon T, Toussaint E, Le Cam J-B, et al (2009) A new characterisation method for rubber. Polymer Testing 28:715-723 . doi: 10.1016/j.polymertesting.2009.06.001 
20. Avril S, Pierron F (2007) General framework for the identification of constitutive parameters from full-field measurements in linear elasticity. International Journal of Solids and Structures 44:49785002 . doi: 10.1016/j.ijsolstr.2006.12.018

21. Pierron F, Vert G, Burguete R, et al (2007) Identification of the orthotropic elastic stiffnesses of composites with the virtual fields method: sensitivity study and experimental validation. Strain 43:250-259

22. Syed-Muhammad K, Toussaint E, Grédiac M, et al (2008) Characterization of composite plates using the virtual fields method with optimized loading conditions. Composite Structures 85:70-82 . doi: 10.1016/j.compstruct.2007.10.021

23. Rossi M, Pierron F (2012) On the use of simulated experiments in designing tests for material characterization from full-field measurements. International Journal of Solids and Structures 49:420-435 . doi: 10.1016/j.ijsolstr.2011.09.025

24. Rossi M, Lava P, Pierron F, et al (2015) Effect of DIC Spatial Resolution, Noise and Interpolation Error on Identification Results with the VFM: Effect of DIC Spatial Resolution, Noise and Interpolation on VFM Identification. Strain 51:206-222 . doi: 10.1111/str.12134

25. Sutton MA, Orteu JJ, Schreier H (2009) Image Correlation for Shape, Motion and Deformation Measurements: Basic Concepts,Theory and Applications. Springer Science \& Business Media

26. Pan B, Qian K, Xie H, Asundi A (2009) Two-dimensional digital image correlation for in-plane displacement and strain measurement: a review. Measurement Science and Technology 20:062001 . doi: 10.1088/0957-0233/20/6/062001

27. Chu TC, Ranson WF, Sutton MA (1985) Applications of digital-image-correlation techniques to experimental mechanics. Experimental mechanics 25:232-244

28. Hijazi A, Friedl A, Kähler CJ (2011) Influence of camera's optical axis non-perpendicularity on measurement accuracy of two-dimensional digital image correlation. JJMIE 5:

29. Siddiqui MZ, Ahmed MF (2014) An Out-of-Plane Motion Compensation Strategy for Improving Material Parameter Estimation Accuracy with 2D Field Measurements. Experimental Mechanics 54:1259-1268 . doi: 10.1007/s11340-014-9880-4

30. Siddiqui MZ (2015) Towards Eliminating the Displacement Bias Due to Out-Of-Plane Motion in 2D Inverse Problems: A Case of General Rigid-Body Motion: 2D-Optical Displacement Field Measurement Methods for Inverse Problems. Strain 51:55-70 . doi: 10.1111/str.12120

31. Yoneyama S, Kitagawa A, Iwata S, et al (2007) Bridge deflection measurement using digital image correlation. Experimental Techniques 31:34-40

32. Hild F, Roux S (2006) Digital image correlation: from displacement measurement to identification of elastic properties-a review. Strain 42:69-80 
33. Sutton MA, Yan JH, Tiwari V, et al (2008) The effect of out-of-plane motion on 2D and 3D digital image correlation measurements. Optics and Lasers in Engineering 46:746-757 . doi:

10.1016/j.optlaseng.2008.05.005

34. Wittevrongel L, Badaloni M, Balcaen R, et al (2015) Evaluation of Methodologies for Compensation of Out of Plane Motions in a 2D Digital Image Correlation Setup: Evaluation of Methodologies for Compensation of Out of Plane Motions in a 2D DIC. Strain 51:357-369 . doi: 10.1111/str.12146

35. Siddiqui MZ, Khan SZ, Khan MA, et al (2017) A Projected Finite Element Update Method for Inverse Identification of Material Constitutive Parameters in transversely Isotropic Laminates.

Experimental Mechanics 57:755-772 . doi: 10.1007/s11340-017-0269-z

36. Szeliski R (2011) Computer Vision. Springer London, London

37. Marsh D (2005) Applied Geometry for Computer Graphics and CAD. Springer-Verlag, London

38. Hartley R, Zisserman A (2003) Multiple view geometry in computer vision, 2nd ed. Cambridge University Press, Cambridge, UK ; New York 


\section{Tables}

Table 1 Geometric parameters for test cases

\begin{tabular}{|c|c|c|c|c|c|c|c|c|c|}
\hline Case ID & $\mathrm{L}$ & $\mathrm{W}$ & $\mathrm{Lx}$ & $\mathrm{Ly}$ & $\alpha$ & $\mathrm{S} 1$ & $\mathrm{~S} 2$ & $\mathrm{~S} 3$ & $\mathrm{~S} 4$ \\
\hline 1 & 185.6 & 137 & 0 & 0 & 0 & 0 & 0 & 0 & 0 \\
\hline 2 & 185.6 & 137 & -0.26 & 0.464 & 0 & 0 & 0 & 0 & \\
\hline 3 & 185.6 & 137 & 0 & 0.5 & 0 & 0 & 0 & 0 & 0 \\
\hline 4 & 185.6 & 137 & -0.41 & -0.28 & 0 & 0.071 & 0 & 0 & -0.76 \\
\hline 5 & 185.6 & 137 & 0 & 0.246 & 0 & 0 & 0 & 0.367 & 0 \\
\hline
\end{tabular}

Table 2 Sensitivity criterion for all test cases

\begin{tabular}{|c|c|c|c|c|c|}
\hline \multirow{2}{*}{ Case ID } & \multicolumn{4}{|c|}{$S c_{\text {rel }}(\%)$} & Max deflection \\
\cline { 2 - 5 } & $E_{1}$ & $E_{2}$ & $v_{12}$ & $G_{12}$ & $(\mathrm{~mm})$ \\
\hline 1 & 13.7 & 74.3 & 3.0 & 9.0 & 0.723 \\
\hline 2 & 37.6 & 23.8 & 2.7 & 36.0 & 0.330 \\
\hline 3 & 65.2 & 5.6 & 3.2 & 26.1 & 0.583 \\
\hline 4 & 10.0 & 38.1 & 1.5 & 50.4 & 0.468 \\
\hline 5 & 31.4 & 32.5 & 2.8 & 33.4 & 0.435 \\
\hline
\end{tabular}

Table 3 Coefficient of variation, relative error and the convergence criterion from simulation results

\begin{tabular}{|c|c|c|c|c|c|c|c|c|c|c|c|c|}
\hline case ID & \multicolumn{3}{|c|}{ Coefficient of variation } & \multicolumn{4}{|c|}{ Relative error } & \multicolumn{3}{|c|}{ Convergence criterion } \\
\hline & $E_{1}$ & $E_{2}$ & $v_{12}$ & $G_{12}$ & $E_{1}$ & $E_{2}$ & $v_{12}$ & $G_{12}$ & $E_{1}$ & $E_{2}$ & $v_{12}$ & $G_{12}$ \\
\hline 1 & 6.5 & 1.6 & 36.7 & 10.7 & 1.1 & 0.8 & 23.0 & 3.0 & 6.6 & 1.8 & 43.3 & 11.1 \\
\hline 2 & 2.6 & 10.3 & 62.9 & 4.4 & 0.4 & 0.6 & 0.9 & 0.8 & 2.6 & 10.3 & 62.9 & 4.5 \\
\hline 3 & 3.0 & 17.3 & 61.6 & 9.6 & 0.4 & 5.8 & 14.5 & 0.8 & 3.0 & 18.2 & 63.3 & 9.6 \\
\hline 4 & 10.6 & 1.6 & 29.7 & 1.6 & 1.7 & 0.7 & 41.2 & 0.9 & 10.7 & 1.7 & 50.7 & 1.9 \\
\hline 5 & 2.9 & 5.0 & 63.1 & 3.9 & 1.6 & 2.3 & 23.8 & 0.9 & 3.3 & 5.5 & 67.5 & 4.0 \\
\hline
\end{tabular}


Table 4 Identification results for case 1

\begin{tabular}{|c|c|c|c|c|c|c|c|c|c|}
\hline \multicolumn{4}{|c|}{ Material Parameters } & \multicolumn{7}{c|}{ Rigid body modes } \\
\hline$E_{1}$ & $E_{2}$ & $v_{12}$ & $G_{12}$ & $T_{x}$ & $T_{y}$ & $\theta_{z}$ & $T_{z}$ & $\theta_{x}$ & $\theta_{y}$ \\
\hline$(\mathrm{MPa})$ & $(\mathrm{MPa})$ & & $(\mathrm{MPa})$ & $(\mathrm{mm})$ & $(\mathrm{mm})$ & $(\mathrm{deg})$ & $(\mathrm{mm})$ & $(\mathrm{deg})$ & $(\mathrm{deg})$ \\
\hline 176.9 & 7.8 & 0.29 & 1.3 & 0.008 & -0.011 & -0.001 & -0.019 & -0.008 & 0.008 \\
\hline 161.0 & 7.8 & 0.27 & 1.3 & 0.008 & -0.018 & -0.001 & -0.023 & -0.013 & 0.010 \\
\hline 171.6 & 7.8 & 0.30 & 1.3 & 0.012 & -0.026 & -0.001 & -0.033 & -0.015 & 0.005 \\
\hline 151.7 & 8.0 & 0.33 & 1.3 & 0.018 & -0.030 & 0.000 & -0.031 & -0.014 & 0.007 \\
\hline 152.8 & 7.9 & 0.38 & 1.3 & 0.021 & -0.037 & 0.000 & -0.032 & -0.017 & 0.000 \\
\hline 137.6 & 7.8 & 0.42 & 1.3 & 0.025 & -0.044 & 0.000 & -0.023 & -0.020 & 0.006 \\
\hline 129.9 & 7.0 & 0.53 & 8.8 & 0.024 & -0.051 & 0.000 & -0.075 & -0.035 & 0.002 \\
\hline 140.4 & 7.1 & 0.53 & 8.8 & 0.026 & -0.053 & 0.000 & -0.090 & -0.028 & 0.004 \\
\hline 126.6 & 7.1 & 0.53 & 8.4 & 0.027 & -0.058 & 0.001 & -0.087 & -0.033 & 0.005 \\
\hline 140.7 & 7.2 & 0.52 & 5.7 & 0.027 & -0.063 & 0.001 & -0.087 & -0.035 & 0.000 \\
\hline
\end{tabular}

Table 5 Identification results for case 2

\begin{tabular}{|c|c|c|c|c|c|c|c|c|c|}
\hline \multicolumn{4}{|c|}{ Material Parameters } & \multicolumn{6}{c|}{ Rigid body modes } \\
\hline$E_{1}$ & $E_{2}$ & $v_{12}$ & $G_{12}$ & $T_{x}$ & $T_{y}$ & $\theta_{z}$ & $T_{z}$ & $\theta_{x}$ & $\theta_{y}$ \\
\hline$(\mathrm{MPa})$ & $(\mathrm{MPa})$ & & $(\mathrm{MPa})$ & $(\mathrm{mm})$ & $(\mathrm{mm})$ & $(\mathrm{deg})$ & $(\mathrm{mm})$ & $(\mathrm{deg})$ & $(\mathrm{deg})$ \\
\hline 123.5 & 6.2 & 0.39 & 6.0 & -0.003 & 0.004 & 0.000 & -0.036 & -0.021 & -0.006 \\
\hline 106.7 & 6.9 & 0.53 & 5.9 & -0.004 & 0.009 & 0.000 & -0.045 & -0.017 & -0.009 \\
\hline 116.9 & 5.8 & 0.53 & 5.9 & -0.006 & 0.017 & 0.000 & -0.061 & -0.022 & -0.011 \\
\hline 120.9 & 6.1 & 0.53 & 6.2 & -0.007 & 0.023 & 0.000 & -0.081 & -0.026 & -0.012 \\
\hline 117.3 & 7.0 & 0.53 & 5.3 & -0.007 & 0.027 & 0.000 & -0.086 & -0.028 & -0.021 \\
\hline 114.7 & 6.0 & 0.53 & 5.2 & -0.009 & 0.031 & 0.000 & -0.090 & -0.027 & -0.017 \\
\hline 119.1 & 6.4 & 0.53 & 5.2 & -0.009 & 0.038 & 0.000 & -0.099 & -0.037 & -0.019 \\
\hline 123.0 & 6.5 & 0.53 & 5.5 & -0.010 & 0.039 & 0.000 & -0.112 & -0.045 & -0.022 \\
\hline 113.4 & 6.6 & 0.53 & 5.5 & -0.010 & 0.044 & 0.000 & -0.121 & -0.040 & -0.024 \\
\hline 120.3 & 7.0 & 0.53 & 5.1 & -0.012 & 0.049 & 0.000 & -0.137 & -0.051 & -0.026 \\
\hline
\end{tabular}


Table 6 Identification results for case 3

\begin{tabular}{|c|c|c|c|c|c|c|c|c|c|}
\hline \multicolumn{4}{|c|}{ Material Parameters } & \multicolumn{7}{c|}{ Rigid body modes } \\
\hline$E_{1}$ & $E_{2}$ & $v_{12}$ & $G_{12}$ & $T_{x}$ & $T_{y}$ & $\theta_{z}$ & $T_{z}$ & $\theta_{x}$ & $\theta_{y}$ \\
\hline$(\mathrm{MPa})$ & $(\mathrm{MPa})$ & & $(\mathrm{MPa})$ & $(\mathrm{mm})$ & $(\mathrm{mm})$ & $(\mathrm{deg})$ & $(\mathrm{mm})$ & $(\mathrm{deg})$ & $(\mathrm{deg})$ \\
\hline 121.1 & 13.9 & 0.08 & 3.5 & 0.000 & 0.009 & -0.001 & -0.036 & -0.009 & 0.002 \\
\hline 130.3 & 14.0 & 0.53 & 2.4 & 0.000 & 0.012 & -0.002 & -0.043 & -0.011 & 0.001 \\
\hline 129.6 & 12.8 & 0.53 & 2.4 & -0.007 & 0.018 & -0.002 & -0.055 & -0.010 & 0.003 \\
\hline 115.6 & 6.9 & 0.08 & 4.1 & -0.005 & 0.021 & -0.002 & -0.064 & -0.012 & 0.000 \\
\hline 120.0 & 8.9 & 0.08 & 3.9 & -0.004 & 0.023 & -0.003 & -0.070 & -0.018 & -0.001 \\
\hline 125.6 & 14.0 & 0.08 & 2.8 & -0.006 & 0.028 & -0.003 & -0.072 & -0.015 & 0.000 \\
\hline 120.0 & 10.3 & 0.08 & 3.7 & -0.007 & 0.031 & -0.004 & -0.089 & -0.020 & -0.001 \\
\hline 123.1 & 10.6 & 0.08 & 3.7 & -0.009 & 0.034 & -0.004 & -0.104 & -0.023 & -0.001 \\
\hline 125.1 & 10.2 & 0.08 & 4.0 & -0.007 & 0.035 & -0.004 & -0.119 & -0.027 & 0.001 \\
\hline 127.1 & 14.0 & 0.08 & 3.0 & -0.008 & 0.034 & -0.005 & -0.117 & -0.018 & -0.001 \\
\hline
\end{tabular}

Table 7 Identification results for case 4

\begin{tabular}{|c|c|c|c|c|c|c|c|c|c|}
\hline \multicolumn{9}{|c|}{ Material Parameters } & \multicolumn{7}{c|}{ Rigid body modes } \\
\hline$E_{1}$ & $E_{2}$ & $v_{12}$ & $G_{12}$ & $T_{x}$ & $T_{y}$ & $\theta_{z}$ & $T_{z}$ & $\theta_{x}$ & $\theta_{y}$ \\
\hline$(\mathrm{MPa})$ & $(\mathrm{MPa})$ & & $(\mathrm{MPa})$ & $(\mathrm{mm})$ & $(\mathrm{mm})$ & $(\mathrm{deg})$ & $(\mathrm{mm})$ & $(\mathrm{deg})$ & $(\mathrm{deg})$ \\
\hline 115.7 & 9.2 & 0.36 & 5.0 & -0.006 & 0.005 & -0.001 & -0.012 & -0.008 & -0.004 \\
\hline 141.6 & 9.2 & 0.34 & 5.2 & -0.009 & 0.004 & -0.001 & -0.027 & -0.004 & -0.007 \\
\hline 108.1 & 8.6 & 0.40 & 4.7 & -0.012 & 0.006 & -0.002 & -0.014 & -0.016 & -0.003 \\
\hline 94.7 & 8.3 & 0.44 & 4.7 & -0.012 & 0.008 & -0.003 & -0.015 & -0.020 & -0.003 \\
\hline 88.0 & 8.1 & 0.46 & 4.6 & -0.015 & 0.011 & -0.003 & -0.014 & -0.022 & -0.002 \\
\hline 69.1 & 7.8 & 0.49 & 4.5 & -0.020 & 0.015 & -0.004 & -0.014 & -0.034 & 0.002 \\
\hline 64.2 & 7.5 & 0.51 & 4.3 & -0.020 & 0.017 & -0.005 & -0.009 & -0.047 & 0.005 \\
\hline 63.2 & 7.4 & 0.51 & 4.4 & -0.019 & 0.019 & -0.005 & -0.013 & -0.049 & 0.007 \\
\hline 59.0 & 7.3 & 0.52 & 4.3 & -0.022 & 0.021 & -0.005 & -0.013 & -0.058 & 0.010 \\
\hline 61.6 & 7.5 & 0.52 & 4.3 & -0.022 & 0.024 & -0.005 & -0.020 & -0.060 & 0.010 \\
\hline
\end{tabular}


Table 8 Identification results for case 5

\begin{tabular}{|c|c|c|c|c|c|c|c|c|c|}
\hline \multicolumn{4}{|c|}{ Material Parameters } & \multicolumn{7}{c|}{ Rigid body modes } \\
\hline$E_{1}$ & $E_{2}$ & $v_{12}$ & $G_{12}$ & $T_{x}$ & $T_{y}$ & $\theta_{z}$ & $T_{z}$ & $\theta_{x}$ & $\theta_{y}$ \\
\hline$(\mathrm{MPa})$ & $(\mathrm{MPa})$ & & $(\mathrm{MPa})$ & $(\mathrm{mm})$ & $(\mathrm{mm})$ & $(\mathrm{deg})$ & $(\mathrm{mm})$ & $(\mathrm{deg})$ & $(\mathrm{deg})$ \\
\hline 139.6 & 7.6 & 0.08 & 5.0 & 0.000 & -0.002 & 0.003 & -0.034 & -0.014 & -0.012 \\
\hline 141.1 & 7.7 & 0.29 & 4.8 & -0.002 & -0.001 & 0.004 & -0.036 & -0.021 & -0.009 \\
\hline 168.2 & 9.0 & 0.08 & 3.5 & -0.005 & 0.002 & 0.006 & -0.041 & -0.009 & -0.009 \\
\hline 146.8 & 7.3 & 0.08 & 4.8 & -0.005 & 0.000 & 0.006 & -0.053 & -0.023 & -0.013 \\
\hline 155.6 & 8.0 & 0.10 & 4.4 & -0.007 & 0.003 & 0.007 & -0.060 & -0.028 & -0.015 \\
\hline 146.2 & 7.8 & 0.08 & 4.4 & -0.006 & 0.005 & 0.008 & -0.061 & -0.027 & -0.016 \\
\hline 150.8 & 8.0 & 0.08 & 4.3 & -0.009 & 0.003 & 0.008 & -0.072 & -0.027 & -0.015 \\
\hline 150.2 & 7.8 & 0.08 & 4.6 & -0.006 & 0.006 & 0.009 & -0.083 & -0.038 & -0.025 \\
\hline 153.8 & 8.2 & 0.08 & 4.2 & -0.008 & 0.006 & 0.009 & -0.091 & -0.036 & -0.020 \\
\hline 149.5 & 7.9 & 0.09 & 4.4 & -0.008 & 0.007 & 0.009 & -0.092 & -0.045 & -0.025 \\
\hline
\end{tabular}

Table 9 Coefficient of variation, relative error and the convergence criterion from experimental results

\begin{tabular}{|c|c|c|c|c|c|c|c|c|c|c|c|c|}
\hline case ID & \multicolumn{4}{|c|}{ Coefficient of variation } & \multicolumn{4}{c|}{ Relative error } & \multicolumn{3}{c|}{ Convergence criterion } \\
\hline & $E_{1}$ & $E_{2}$ & $v_{12}$ & $G_{12}$ & $E_{1}$ & $E_{2}$ & $v_{12}$ & $G_{12}$ & $E_{1}$ & $E_{2}$ & $v_{12}$ & $G_{12}$ \\
\hline 1 & 11 & 5 & 26 & 90 & 3 & 6 & 32 & 22 & 12 & 8 & 42 & 93 \\
\hline 2 & 4 & 6 & 8 & 7 & 19 & 19 & 65 & 12 & 19 & 20 & 66 & 14 \\
\hline 3 & 4 & 22 & 115 & 19 & 15 & 44 & 47 & 33 & 15 & 50 & 124 & 38 \\
\hline 4 & 33 & 9 & 15 & 7 & 40 & 1 & 47 & 8 & 52 & 9 & 49 & 11 \\
\hline 5 & 5 & 6 & 66 & 9 & 4 & 1 & 67 & 11 & 6 & 6 & 94 & 15 \\
\hline
\end{tabular}




\section{Figures}
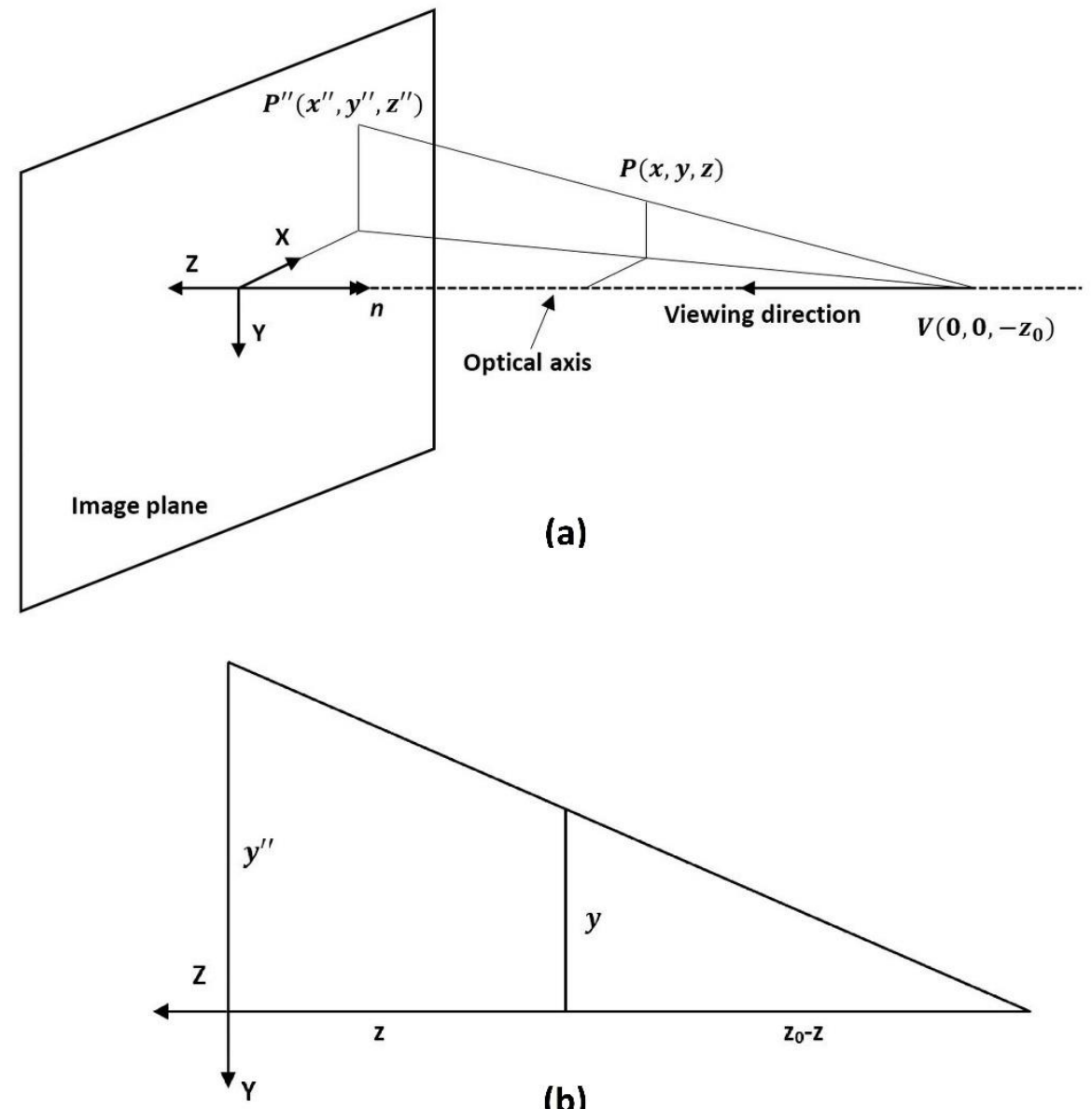

(b)

Figure 1 (a) Perspective projection of a point in space, (b) YZ plane view of (a) 


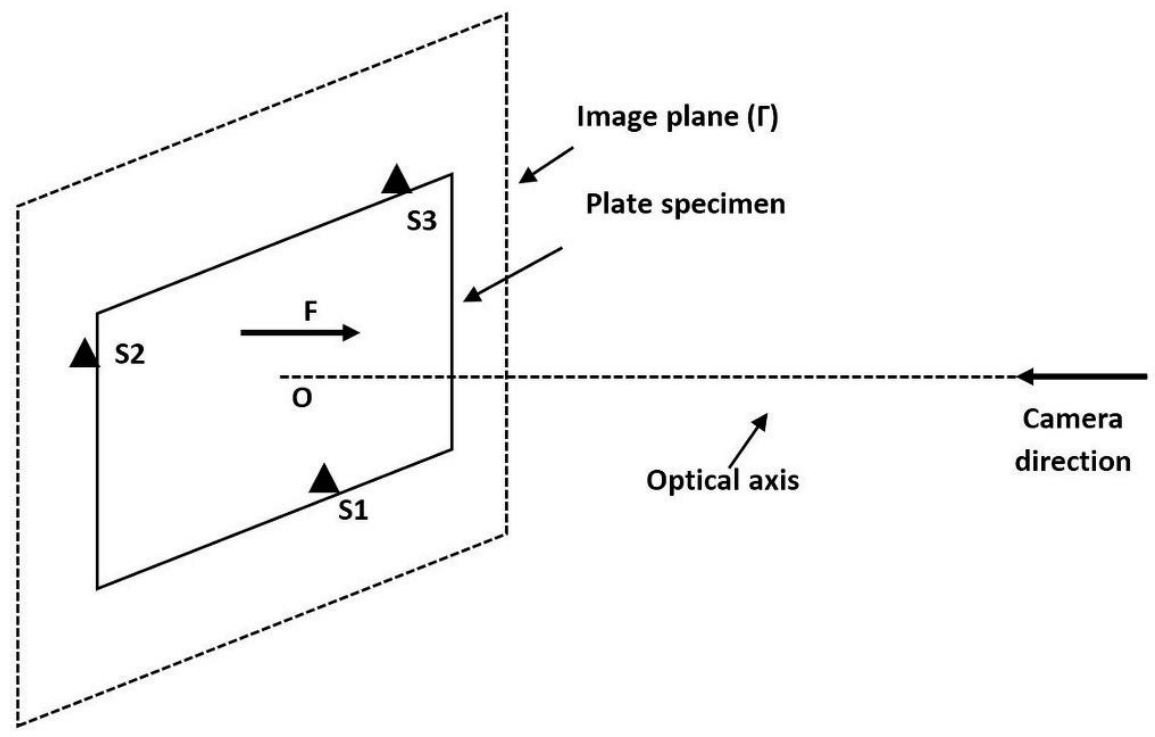

Figure 2 Plate specimen arrangement for inverse identification with PFEUM technique 


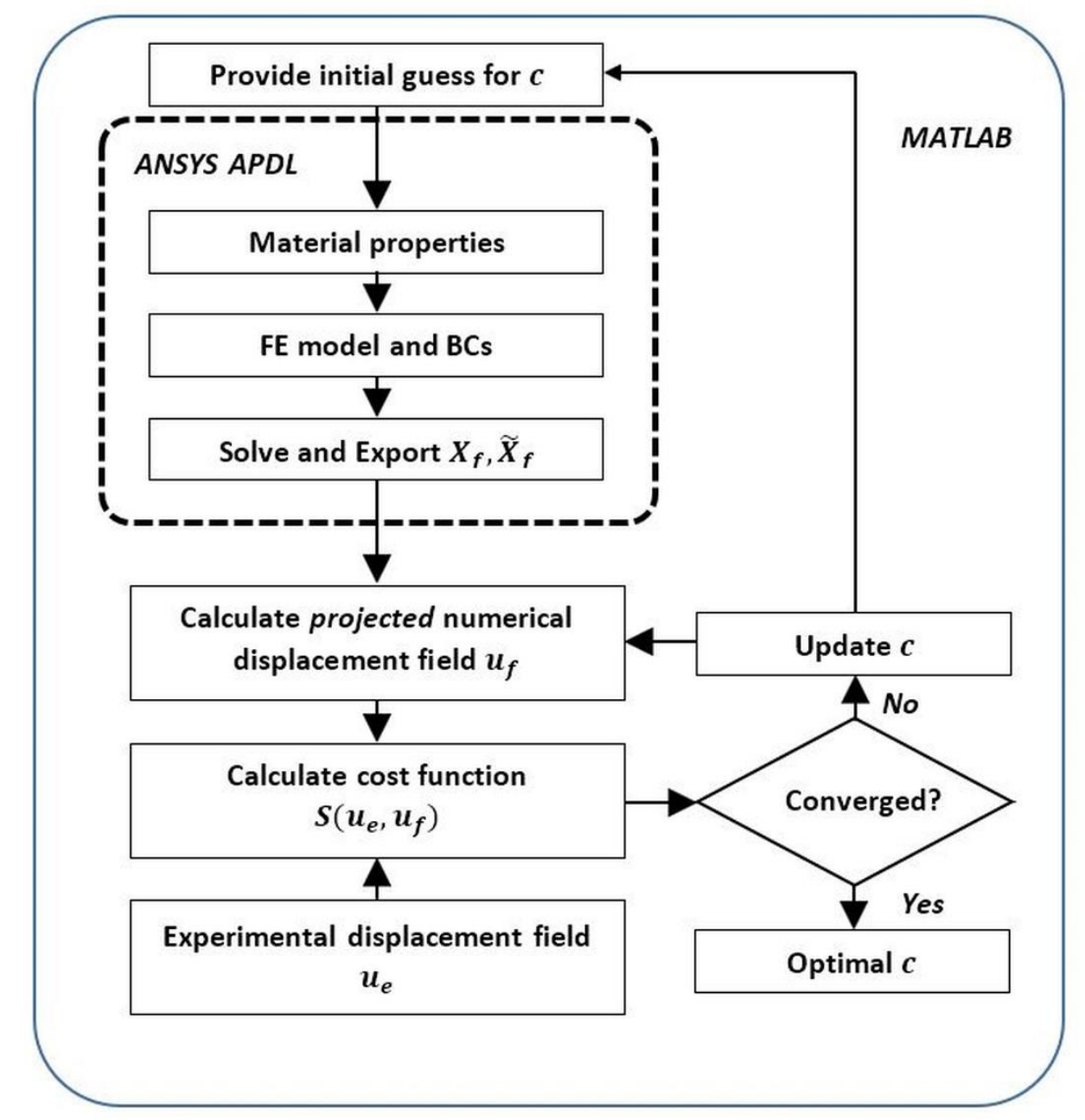

Figure 3 Schematic of inverse identification with PFEUM technique 


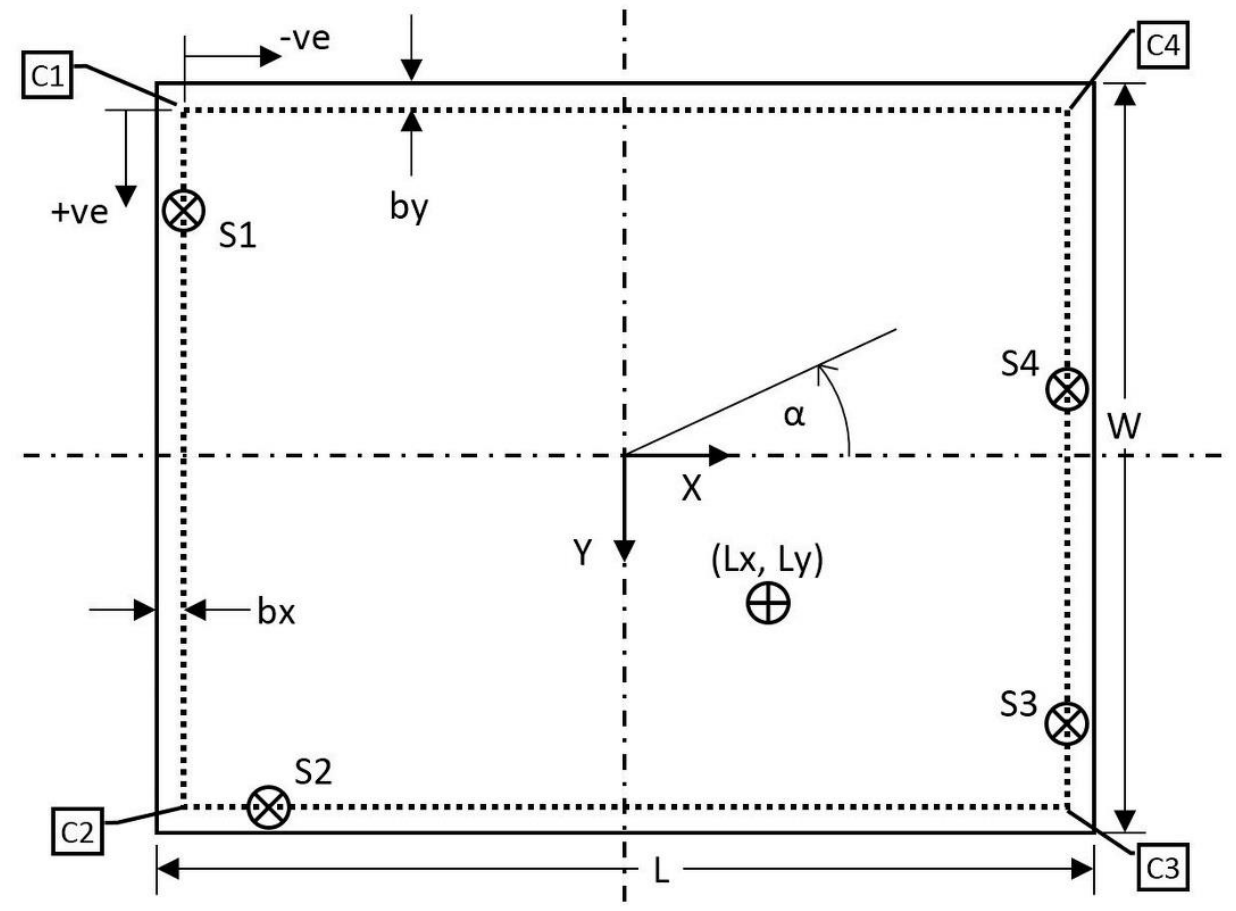

Figure 4 Parametric description of specimen geometry 


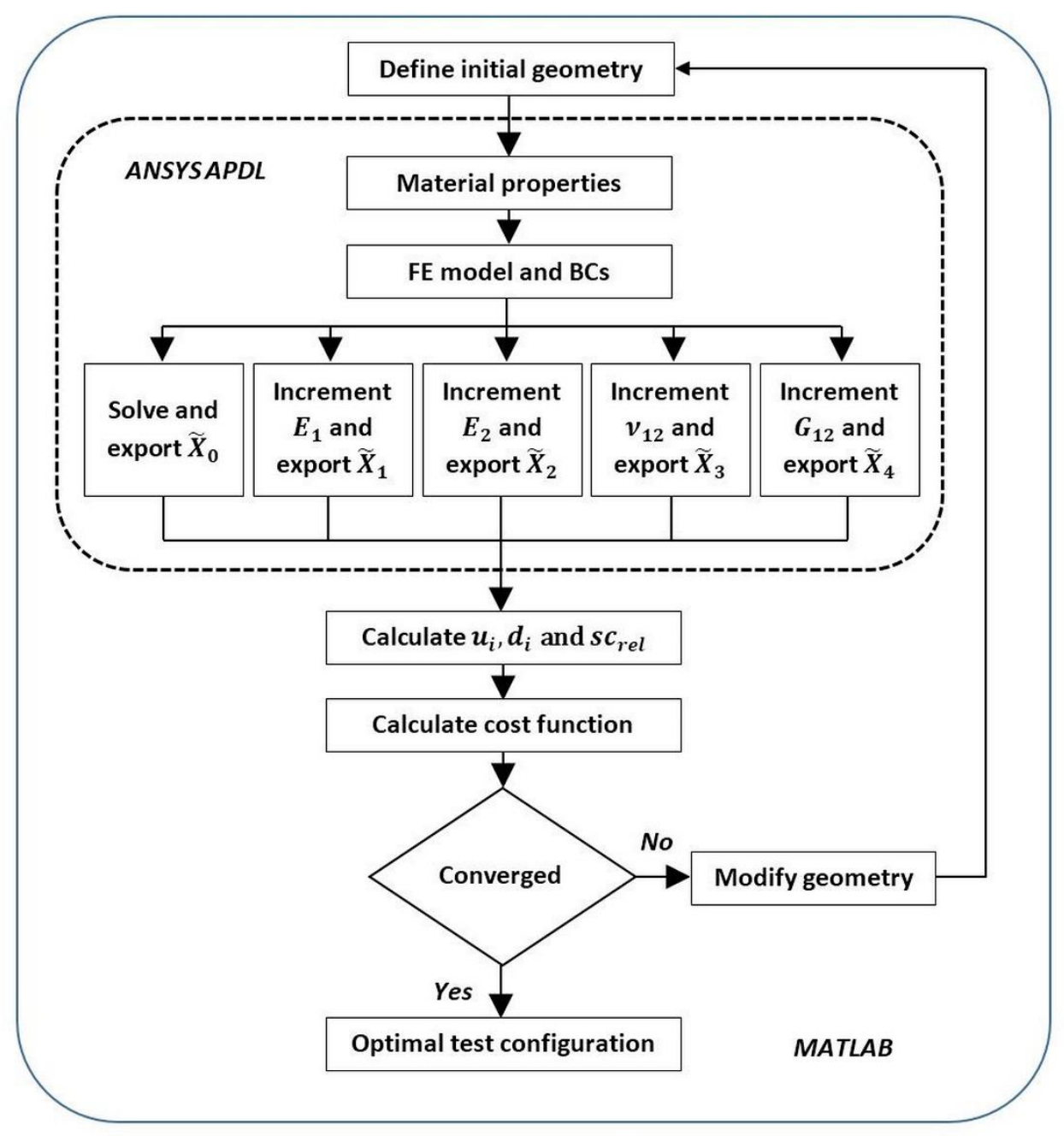

Figure 5 Schematic of sensitivity based test configuration design

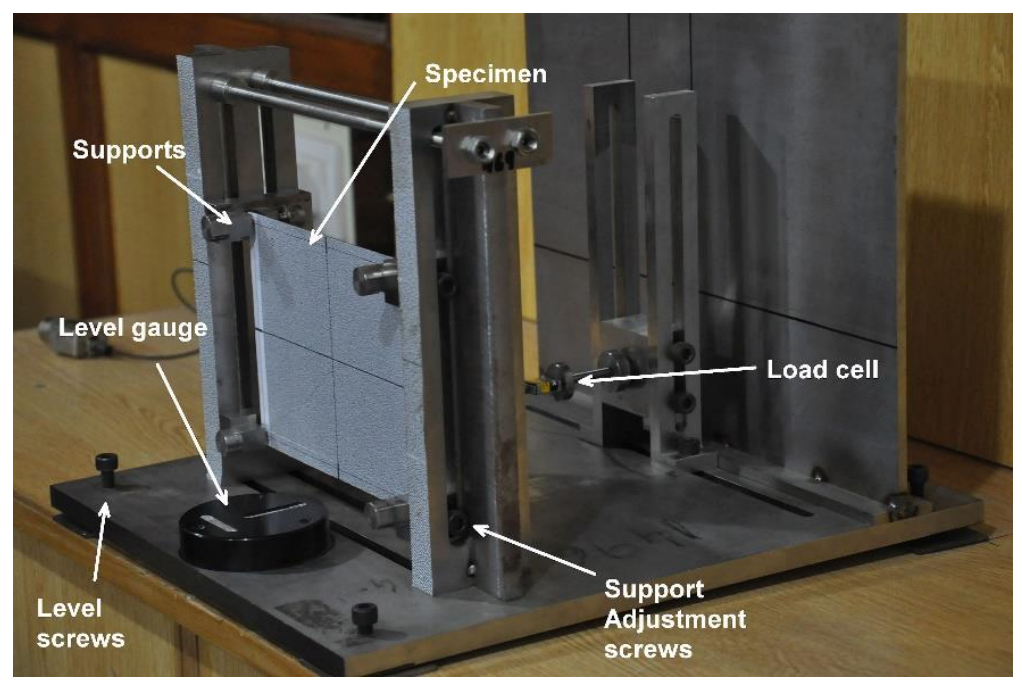

Figure 6 Redesigned experimental setup for inverse identification 


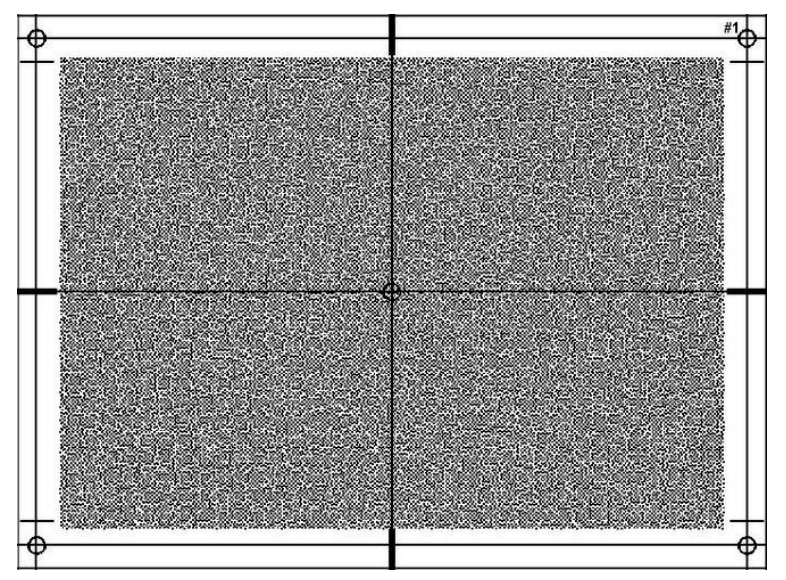

Figure 7 Speckle pattern for DIC measurements

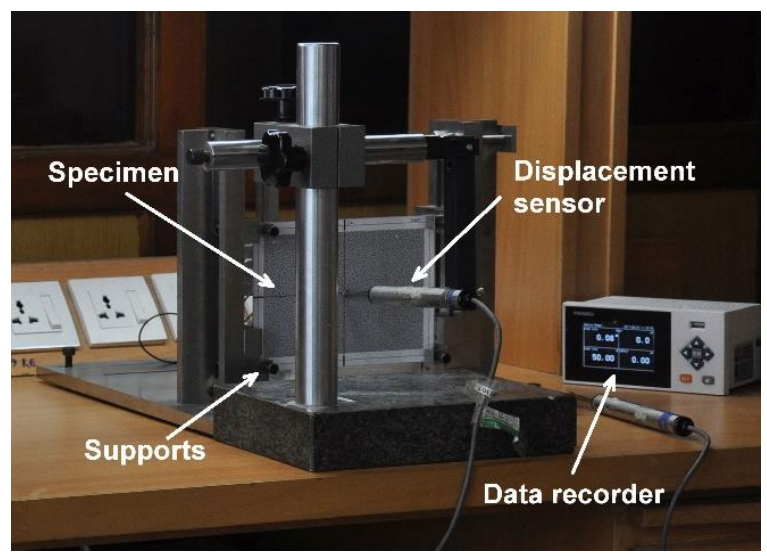

Figure 8 Experimental setup for plate deflection measurement 


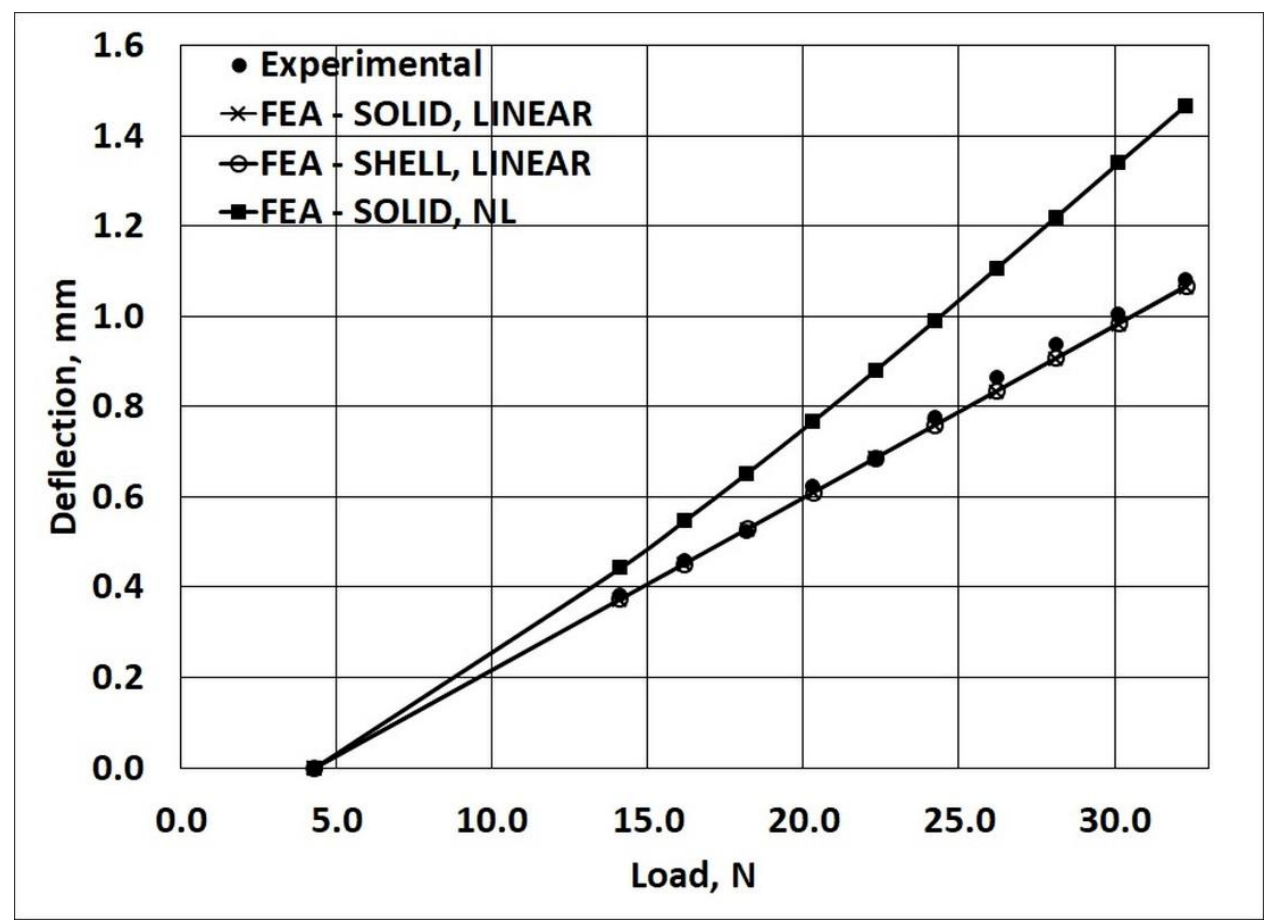

Figure 9 Comparison of computed and measured plate deflection

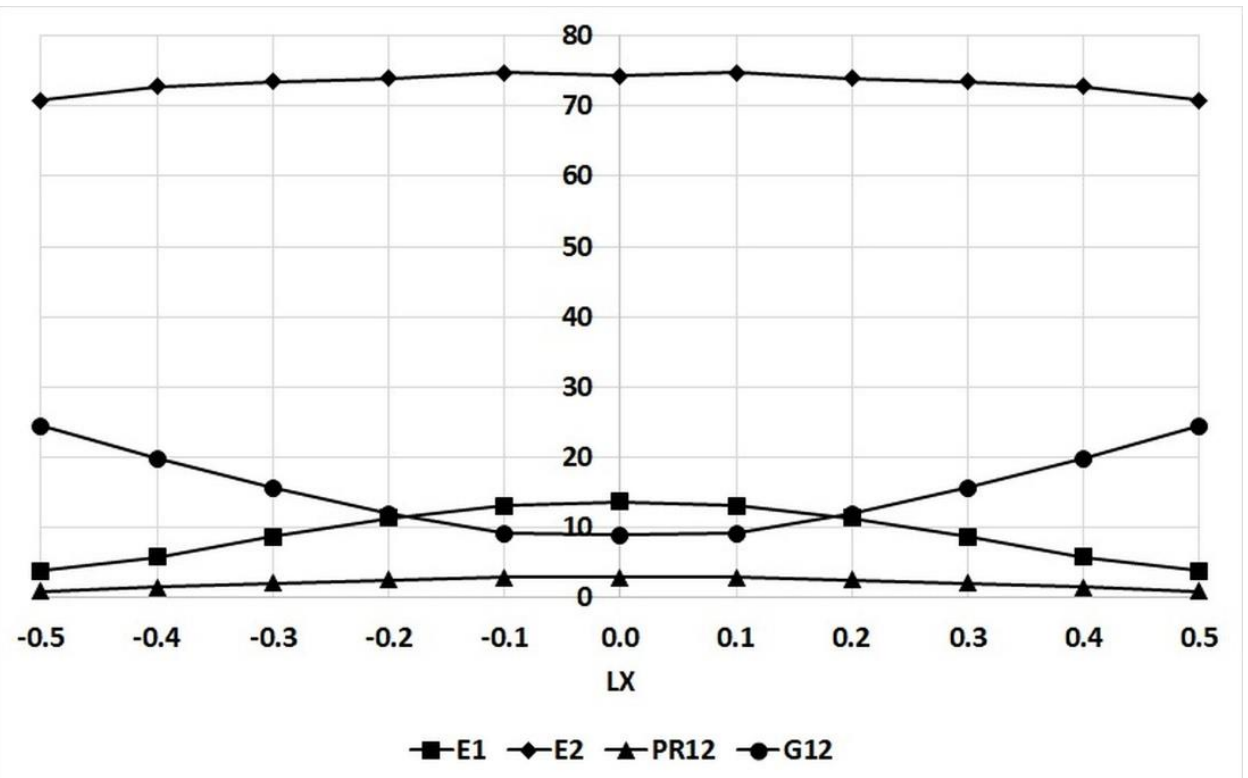

Figure 10 Percent contribution of material parameters as a function of LX varying along plate center 


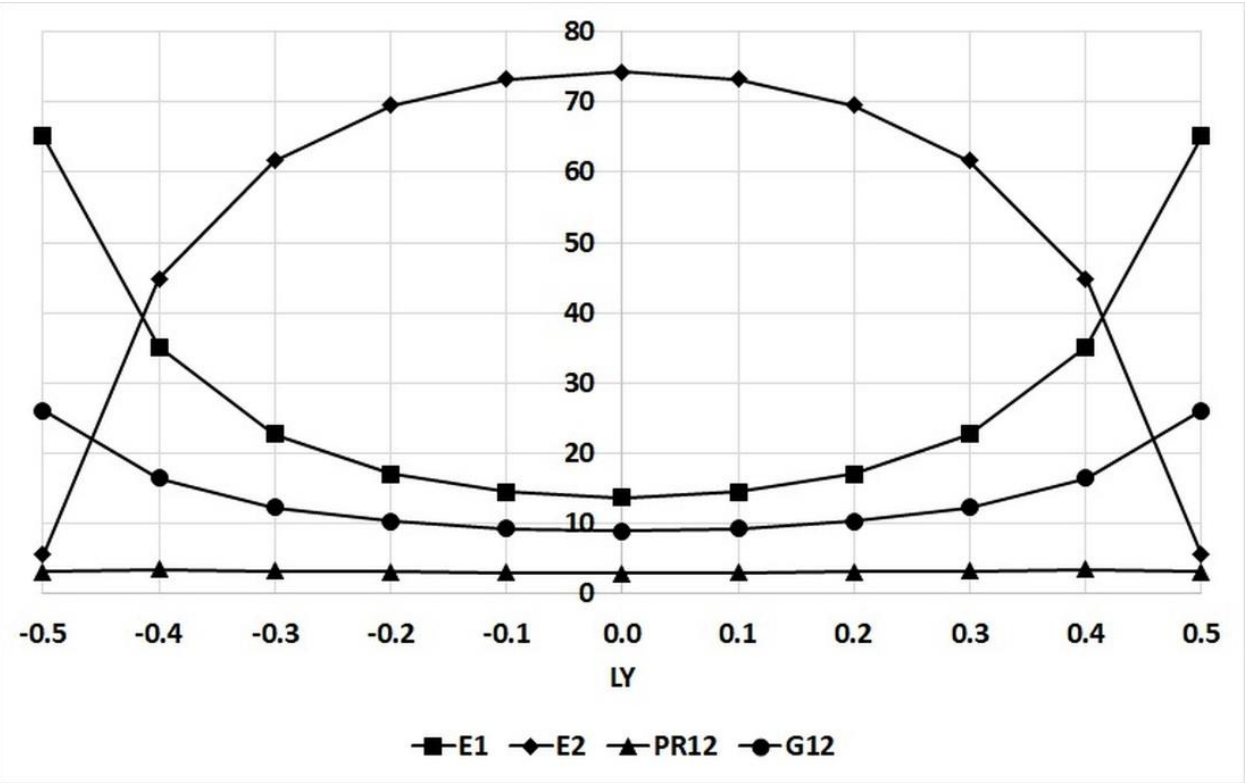

Figure 11 Percent contribution of material parameters as a function of $L Y$ varying along plate center

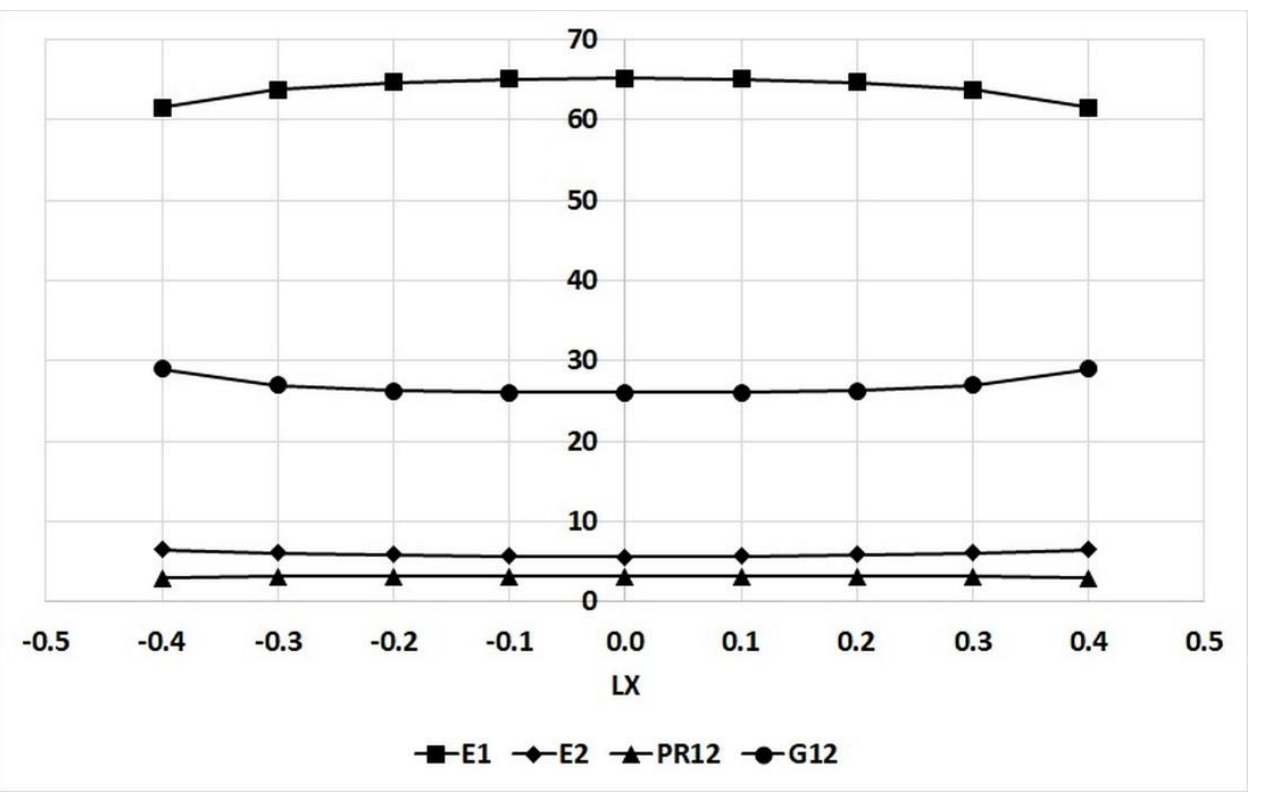

Figure 12 Percent contribution of material parameters as a function of $L X$ varying along bottom edge 


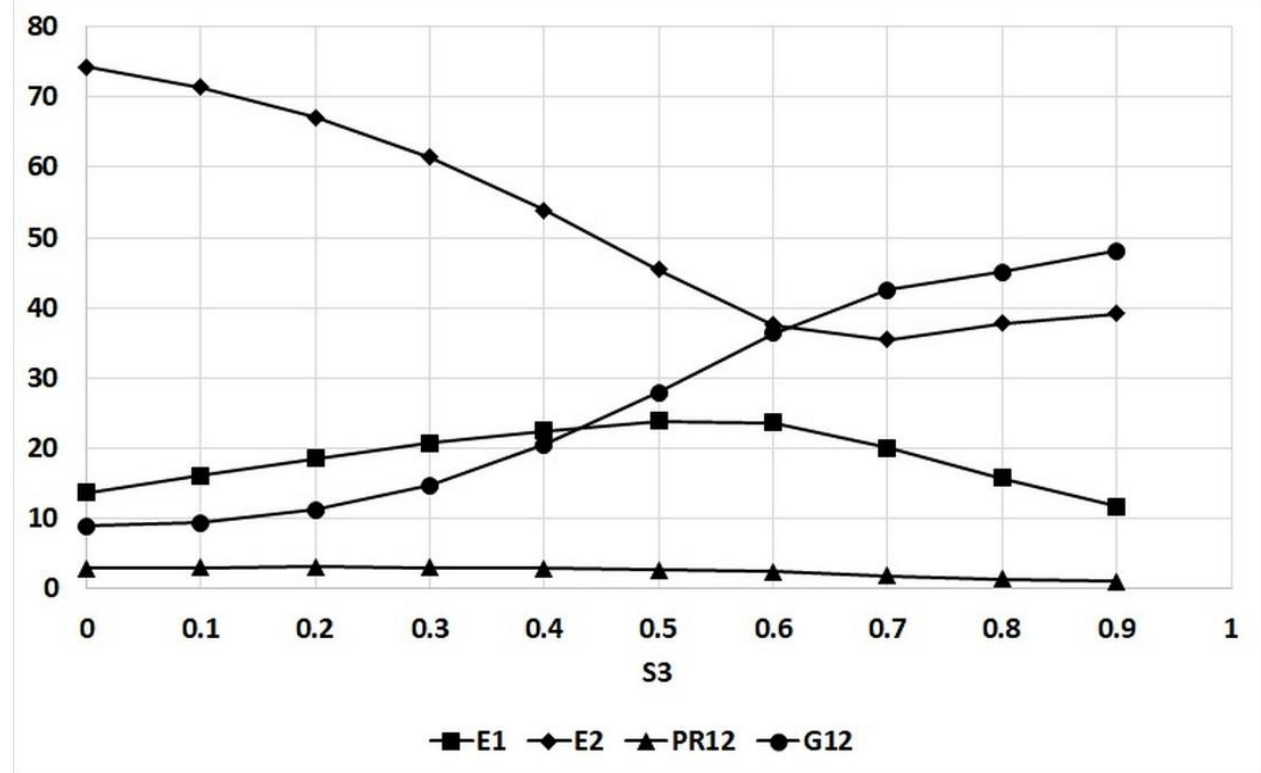

Figure 13 Percent contribution of material parameters as a function of S3 varying along right edge

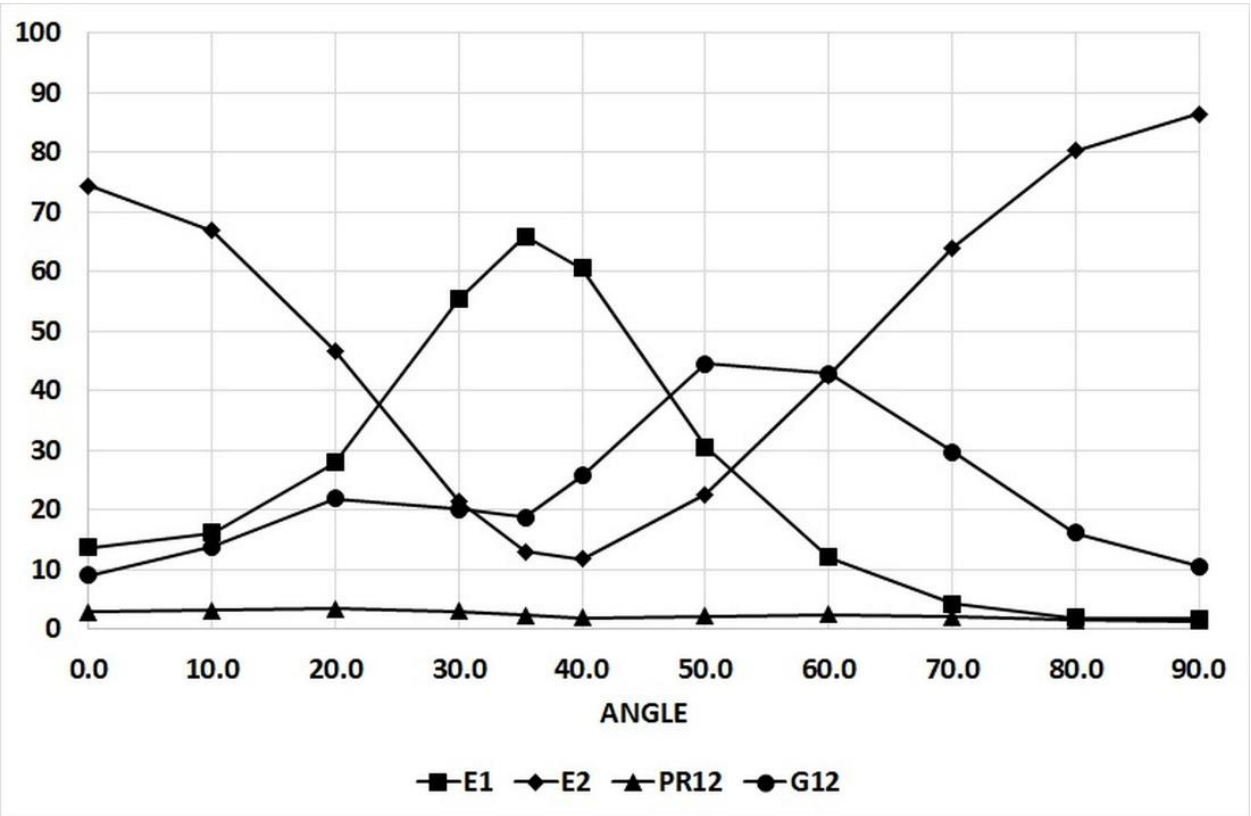

Figure 14 Percent contribution of material parameters as a function of fiber orientation 


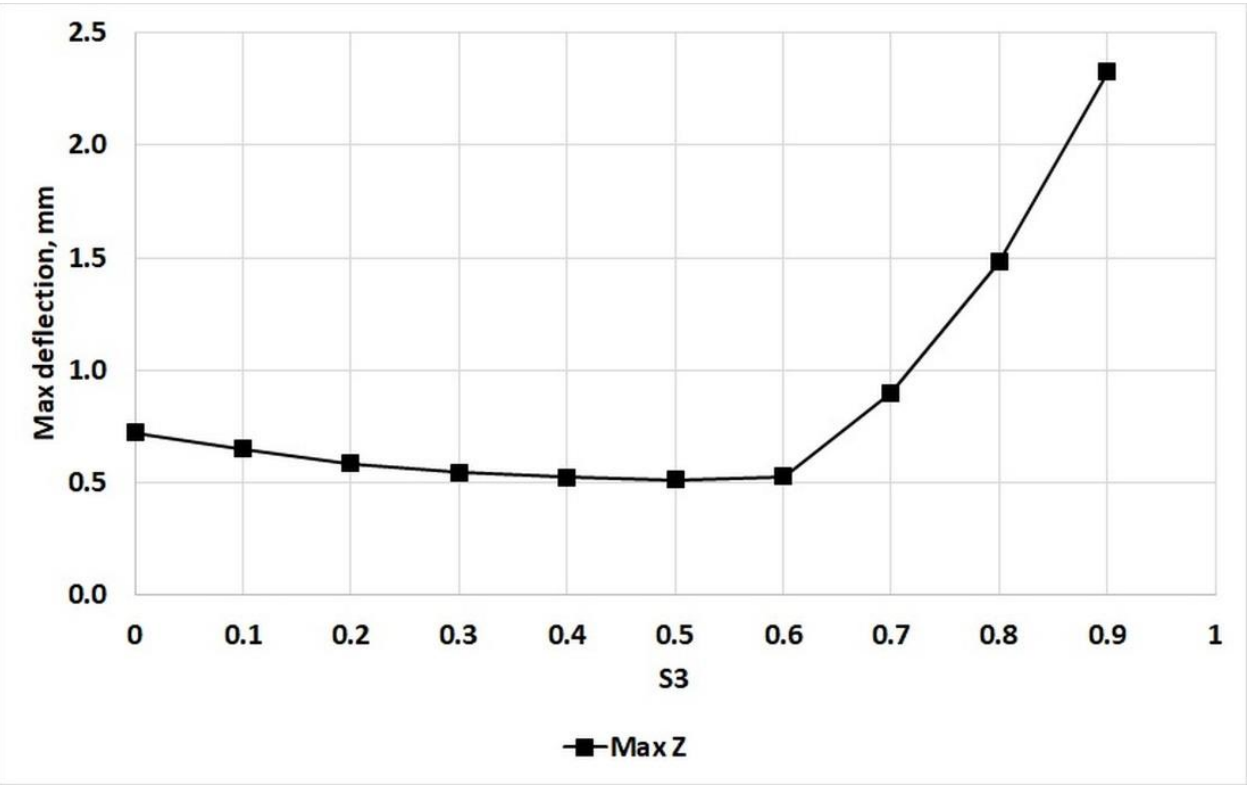

Figure 15 Maximum plate deflection as a function of S3 varying along right edge

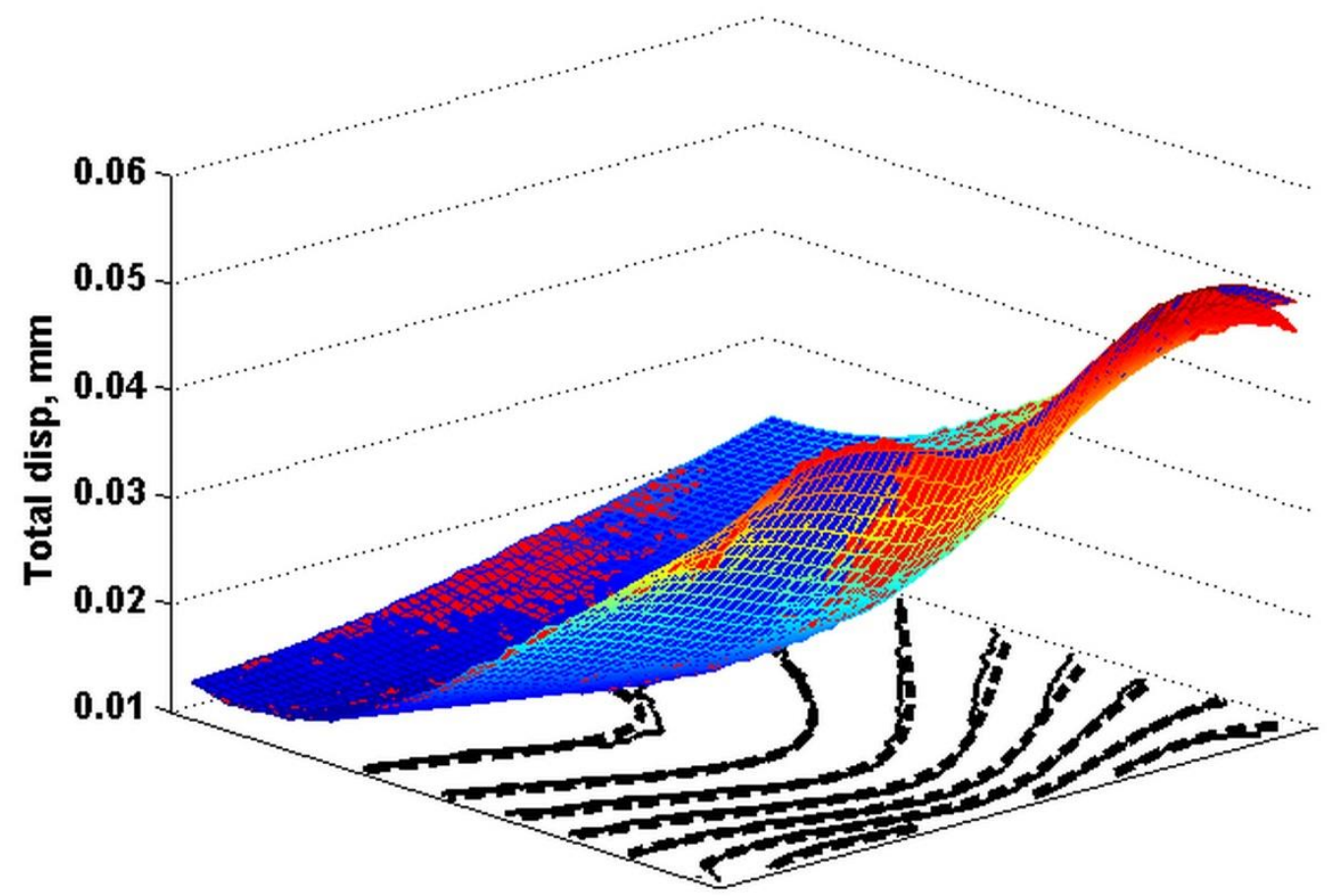

Figure 16 Comparison of numerical (blue, dotted) and experimental (red, solid) projected displacement fields 


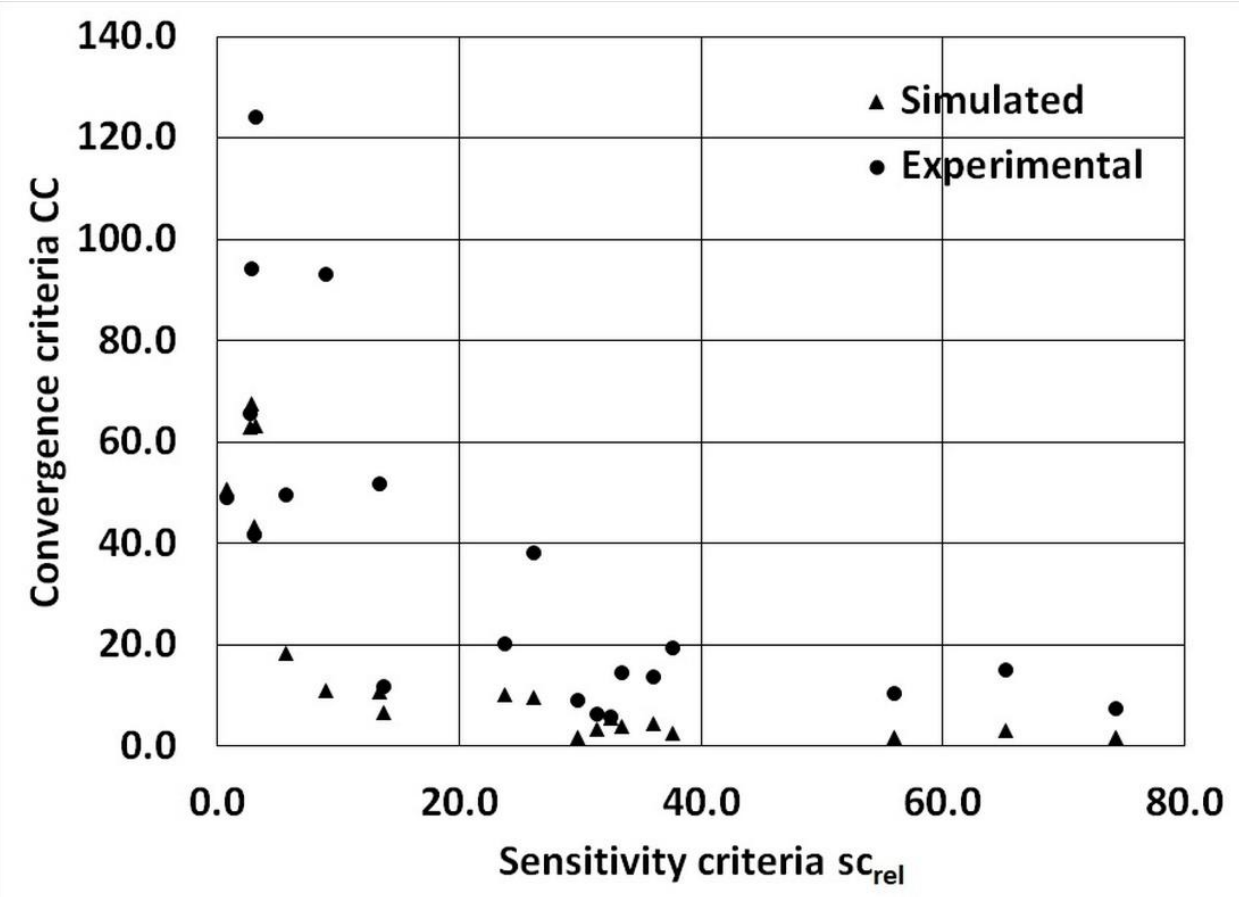

Figure 17 Relationship between parameter convergence with sensitivity criterion for simulated and experimental results

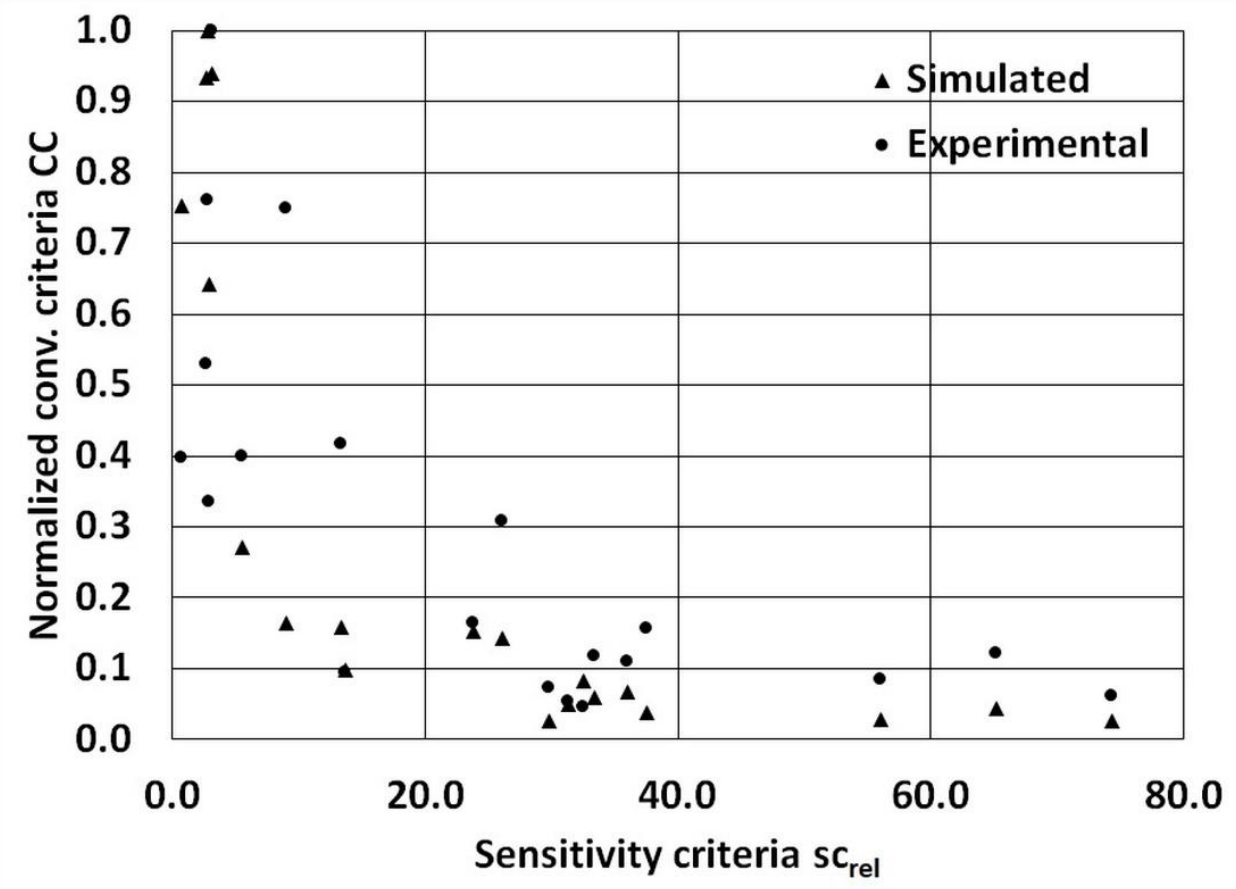

Figure 18 Comparison of simulated and experimental results with normalized convergence criteria 


\section{A novel test configuration design method for inverse identification of in-plane moduli of a composite plate under the PFEUM framework}

Siddiqui, Muhammad Zeeshan

Wiley

Siddiqui MZ, Khan SZ, Khan MA, Shahzad M, Khan KA \& Nisar S. A novel test configuration design method for inverse identification of in-plane moduli of a composite plate under the PFEUM framework. Strain, Volume 54, Issue 5, 2018, Article number e12280

https://doi.org/10.1111/str.12280

Downloaded from Cranfield Library Services E-Repository 